\title{
Reorganization and Movement of Microtubules in Axonal Growth Cones and Developing Interstitial Branches
}

\author{
Erik W. Dent, ${ }^{1}$ John L. Callaway, ${ }^{2}$ Györgyi Szebenyi, ${ }^{2}$ Peter W. Baas, ${ }^{1,2}$ and Katherine Kalil ${ }^{1,2}$ \\ ${ }^{1}$ Neuroscience Training Program and ${ }^{2}$ Department of Anatomy, University of Wisconsin, Madison, Wisconsin 53706
}

Local changes in microtubule organization and distribution are required for the axon to grow and navigate appropriately; however, little is known about how microtubules (MTs) reorganize during directed axon outgrowth. We have used time-lapse digital imaging of developing cortical neurons microinjected with fluorescently labeled tubulin to follow the movements of individual MTs in two regions of the axon where directed growth occurs: the terminal growth cone and the developing interstitial branch. In both regions, transitions from quiescent to growth states were accompanied by reorganization of MTs from looped or bundled arrays to dispersed arrays and fragmentation of long MTs into short MTs. We also found that long-term redistribution of MTs accompanied the withdrawal of some axonal processes and the growth and stabilization of others. Individual MTs moved independently in both anterograde and retrograde directions to explore developing processes. Their velocities were inversely proportional to their lengths. Our results demonstrate directly that MTs move within axonal growth cones and developing interstitial branches. Our findings also provide the first direct evidence that similar reorganization and movement of individual MTs occur in the two regions of the axon where directed outgrowth occurs. These results suggest a model whereby short exploratory MTs could direct axonal growth cones and interstitial branches toward appropriate locations.

Key words: microtubule; interstitial axon branch; growth cone; time-lapse fluorescent microscopy; cortical neuronal culture; cortical development; axon outgrowth
The growing axon contains a dense array of microtubules (MTs) that are individually short relative to the length of the axon but are tightly coalesced into a continuous bundle. MTs are essential for architectural support and also act as railways for the transport of various materials along the length of the axon. During growth and navigation of the axon, the MT array within the growth cone reorganizes and reorients toward the future direction of axon outgrowth (Sabry et al., 1991; Tanaka and Kirschner, 1991; Lin and Forscher, 1993; Tanaka and Sabry, 1995; Tanaka et al., 1995; Letourneau, 1996; Suter et al., 1998). However, the role that MTs play in axon growth is not limited to their continuous rearrangement at the terminal growth cone. In many neural systems, such as those arising from the cerebral cortex, directed axon growth is also accomplished by the formation of collateral branches, which extend interstitially from the axon shaft (O'Leary and Terashima, 1988; Halloran and Kalil, 1994; Kuang and Kalil, 1994; Bastmeyer and O'Leary, 1996). In the vicinity of target regions in which interstitial axon branches develop, growth cones of cortical neurons undergo prolonged pausing behaviors (Halloran and Kalil, 1994; Yamamoto et al., 1997). During pausing periods, cortical growth cones enlarge and reorganize, leaving filopodial and lamellar protrusions along the axon shaft from which interstitial

Received April 16, 1999; revised July 27, 1999; accepted Aug. 2, 1999.

This work was supported by National Institutes of Health Grants NS 14428 to K.K. and NS 34270 to P.W.B. and K.K. and a predoctoral training grant award GM07507 to E.W.D. We thank Dr. Lotfi Ferhat and Dr. Wenqian Yu for encouragement and helpful discussions/comments on this manuscript. We also thank Dr. Fridoon Ahmad, Matthew Schwei, Dr. Bao Xi Gao, and Dr. Lea Ziskind-Conhaim for technical help and encouragement at the beginning of these studies. Movies of several figures can be viewed at http://kalil.anatomy.wisc.edu.

Correspondence should be addressed to Dr. Katherine Kalil, University of Wisconsin, Department of Anatomy, 1300 University Avenue, Madison, WI 53706.

Dr. Szebenyi's present address: Department of Cell Biology and Neuroscience, University of Texas Southwestern Medical Center, 6000 Harry Hines Boulevard, Dallas, TX 75235-9111.

Copyright (C) 1999 Society for Neuroscience $\quad 0270-6474 / 99 / 198894-15 \$ 05.00 / 0$ branches later emerge (Szebenyi et al., 1998). Studies on cultured hippocampal neurons suggest that MTs fragment within the region of the axon where interstitial branches form (Yu et al., 1994). However, little is known about how the MT array reorganizes during the formation of such branches.

What are the specific changes that occur in the MT array within the terminal growth cone and at sites of interstitial branch formation? MTs are polar structures that undergo dynamic assembly and disassembly events (Desai and Mitchison, 1997). In the axon, MTs are oriented with their plus-ends distal to the cell body (Heidemann et al., 1981), and it appears that all assembly and disassembly events occur from the plus-end of the MT (Baas and Ahmad, 1992). Some authors have argued that the reorganization of the MT array is based solely on the assembly and disassembly of MTs and not on their movement through the cytoplasm (for review, see Hirokawa et al., 1997), whereas other authors have argued that individual MTs can interact with motor proteins that actively transport them to new locations (for review, see Baas, 1997; Baas and Brown, 1997). To date, this issue remains controversial, in large part because of results based on relatively lowresolution fluorescence analyses. In these studies, a mass of MTs was marked by photobleaching or photoactivation, but no movement of the mass was observed (Okabe and Hirokawa, 1992, 1993; Sabry et al., 1995; Takeda et al., 1995; Funakoshi et al., 1996; Chang et al., 1998). Nevertheless there is compelling evidence from indirect studies that individual MTs are capable of movement (Yu et al., 1996; Slaughter et al., 1997; Gallo and Letourneau, 1999).

Therefore, to understand how MTs influence directed axon outgrowth at the terminal growth cone and at axon branch points, we focused first on how the bundled MT array is reorganized during transitions from quiescent to growth states and second on whether individual MTs in these regions are capable of indepen- 

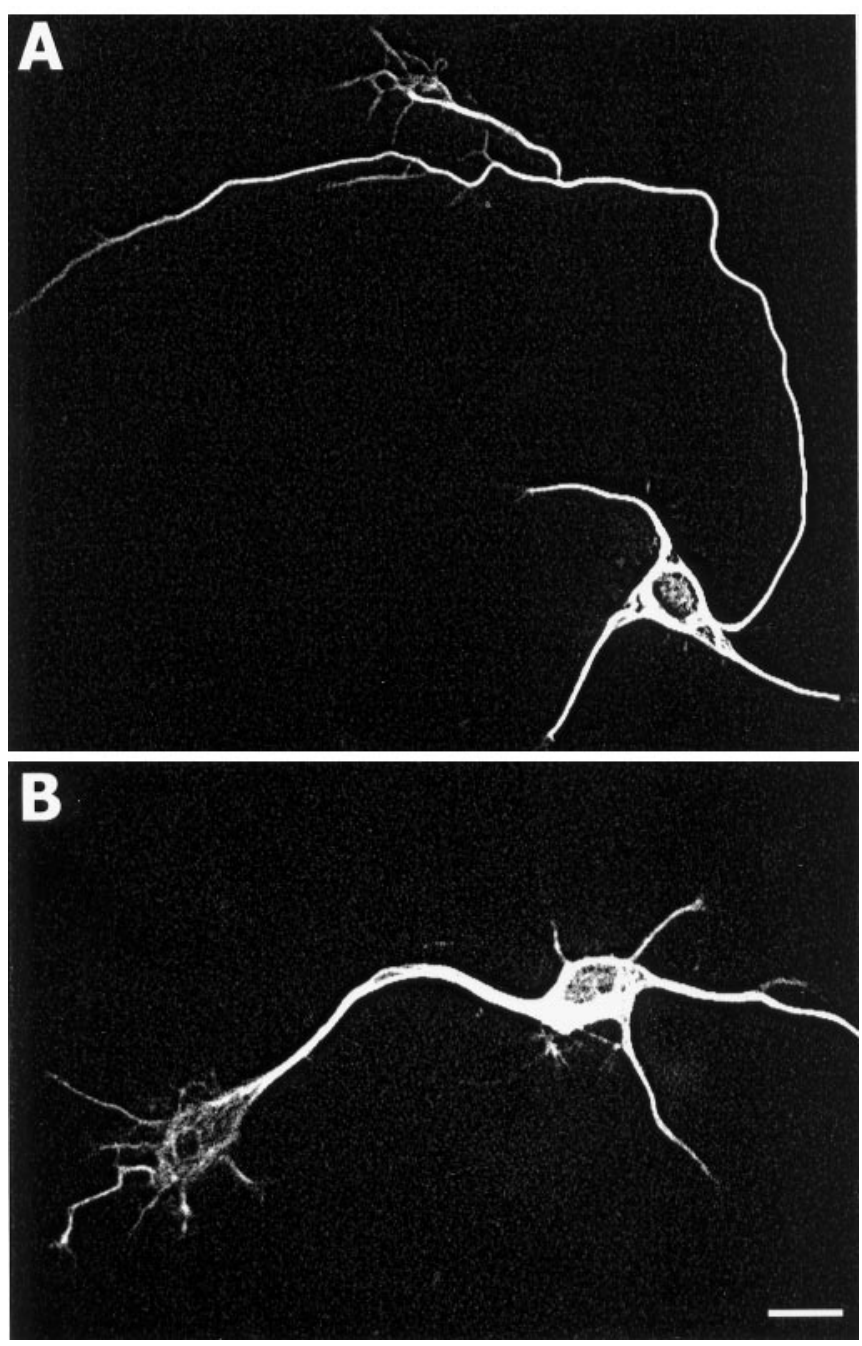

Figure 1. Examples of living cortical neurons microinjected with fluorescently labeled tubulin. By $1 \mathrm{hr}$ after injection, tubulin has diffused throughout the neurons into the minor processes, the single long axons, and an interstitial axon branch $(A)$. Incorporation of tubulin into MTs is demonstrated by the looped arrays within the large axonal growth cones in $A$ and $B$. Scale bar, $20 \mu \mathrm{m}$.

dent movement. We chose for analysis pyramidal neurons from early postnatal sensorimotor cortex because in vivo efferent cortical axons branch interstitially to cortical and subcortical targets (O'Leary and Terashima, 1988). Moreover, in a previous study (Szebenyi et al., 1998) we found that terminal growth cones of cortical axons undergo lengthy pausing behaviors before reorganizing into interstitial axon branches. Therefore, in the present study we examined terminal growth cones as well as axon branches to determine how MTs rearrange from a bundled array to a configuration that would permit new growth. To visualize directly the movements of individual MTs, we used highresolution time-lapse fluorescent digital imaging of dissociated cortical neurons microinjected with fluorescently labeled tubulin.

\section{MATERIALS AND METHODS}

Reagents and culture media. All reagents were purchased from GibcoBRL (Grand Island, NY) unless specified otherwise. Five different media preparations were used in the experiments. The formulations are as follows: dissection medium (Hibernate A with B27 supplement, 0.3\% glucose, $1 \mathrm{~mm}$ L-glutamine, and $10 \mu \mathrm{m}$ gentimycin sulfate), serumcontaining medium [Neurobasal medium with 5\% FBS (Hyclone, Logan,
UT), B27 supplement, $0.3 \%$ glucose, $1 \mathrm{~mL}$-glutamine, and $10 \mu \mathrm{M}$ gentimycin sulfate; final osmolality adjusted to $300-310 \mathrm{mOsm}$ ), serum-free medium (Neurobasal medium with B27 supplement, $0.3 \%$ glucose, $1 \mathrm{~mm}$ L-glutamine, and $10 \mu \mathrm{M}$ gentimycin sulfate; final osmolality adjusted to 300-310 mOsm), imaging medium-1 [degassed, nitrogen-saturated dissection medium with $100 \mu \mathrm{g} / \mathrm{ml}$ sodium pyruvate (Sigma, St. Louis, MO), $1 \mathrm{~mm}$ ascorbic acid (Sigma), $4 \mu \mathrm{g} / \mathrm{ml} \alpha$-tocopherol (Sigma), $12.5 \mu \mathrm{g} / \mathrm{ml}$ catalase (Sigma), $4 \mu \mathrm{g} / \mathrm{ml}$ glutathione (Sigma)] (Brewer and Cotman, 1989; Mikhailov and Gundersen, 1995), and imaging medium-2 [dissection medium that was incubated for 5 min with a 1:100 dilution of Oxyrase (Mansfield, $\mathrm{OH}$ ) at $37^{\circ} \mathrm{C}$ (Waterman-Storer et al., 1993; Mikhailov and Gundersen, 1995), filtered through a $0.22 \mu \mathrm{m}$ filter (Nalge Nunc International, Rochester, NY) and stored under nitrogen].

Cell culture. Cultures were prepared from cortical tissue obtained from the brains of 1- to 3-d-old Syrian golden hamsters (Mesocricetus auratus). The day of birth was considered postnatal day 0 . Pups were anesthetized on ice and decapitated. The entire brain was removed and immediately transferred to ice-cold dissection medium. The sensorimotor cortex was dissected away from the rest of the brain, stripped of meninges, and cut into small pieces with a tungsten needle (Fine Science Tools, Foster City, CA). Cortical pieces were washed twice with $\mathrm{HBSS}$ without $\mathrm{Ca}^{+2} / \mathrm{Mg}^{+2}$ and digested in HBSS without $\mathrm{Ca}^{+2} / \mathrm{Mg}^{+2}$ with $0.025 \%$ trypsin and $0.05 \%$ DNase I (Sigma) for $15 \mathrm{~min}$ at $37^{\circ} \mathrm{C}$. Enzymatically digested cortical pieces were washed twice in serum-containing medium, dissociated by trituration, and centrifuged at $200 \mathrm{rpm}(6 \times g)$ for $5 \mathrm{~min}$. Pellets were resuspended in serum-containing medium and plated at a density of $1000-5000$ cells $/ \mathrm{cm}^{2}$ on etched grid coverslips (Bellco, Vineland, NJ) that had been attached to $35 \mathrm{~mm}$ culture dishes (Corning, Corning, NY) with Valap (Goslin and Banker, 1991). Etched grid coverslips were used so that injected cells could be located for imaging. The coverslips were coated with $0.5 \mathrm{mg} / \mathrm{ml}$ poly-D-lysine (Sigma) in borate buffer and either $20 \mu \mathrm{g} / \mathrm{ml} \mathrm{laminin} \mathrm{(Gibco)} \mathrm{or} 50 \mu \mathrm{g} / \mathrm{ml}$ concanavalin-A (Sigma) in Neurobasal medium. Cells were incubated at $37^{\circ} \mathrm{C} / 5 \% \mathrm{CO}_{2}$. After $2-4 \mathrm{hr}$ a $10 \times$ vol of serum-free medium was added to the cultures. Under these culture conditions, cortical neurons remained viable for 5-7 d and developed a polarity similar to cultured hippocampal neurons (Dotti et al., 1988), with a single long axon and several minor processes (de Lima et al., 1997). Cultures contained very few glial cells $(<10 \%)$.

Preparation and labeling of tubulin. Tubulin was prepared from bovine brain by several cycles of polymerization-depolymerization and stored at $-80^{\circ} \mathrm{C}$ (Hyman et al., 1991). The fluorescent dye 5- $(+6)$ carboxytetramethyl-rhodamine succinimidyl ester (TMR) (Molecular Probes, Eugene, OR) was coupled to tubulin following the procedures outlined in Keating et al. (1997). This labeling procedure resulted in polymerization-competent TMR-tubulin with dye-to-protein ratios of 0.8-1.2. TMR-tubulin used for injections was diluted to $4 \mathrm{mg} / \mathrm{ml}$ for short-term studies and $10 \mathrm{mg} / \mathrm{ml}$ for long-term studies in injection buffer (100 mM PIPES, $0.5 \mathrm{~mm} \mathrm{MgCl}_{2}, \mathrm{pH}$ 6.9), aliquoted, and stored in liquid nitrogen. Before injection, aliquots were thawed and centrifuged at $21,000 \times g(15,000 \mathrm{rpm})$ at $4^{\circ} \mathrm{C}$ for $5 \mathrm{~min}$ (Eppendorf Model 5402, Hamburg, Germany) to remove tubulin aggregates.

Injection of cultured cortical neurons. Pyramidal neurons $15-20 \mu \mathrm{m}$ in diameter were chosen for injection. The osmolality of the culture media was increased to $300-310$ mOsm to minimize osmotic shock to neurons during injection. Dishes were placed on the stage of an Axiovert $135 \mathrm{M}$ inverted microscope (Carl Zeiss, Thornwood, NY), and cells were located under differential interference contrast (DIC) illumination with a $40 \times$ Plan-Neofluor/1.3 NA objective and long-working distance condenser. Filament-containing thin-walled glass pipettes with a $1.0 \mathrm{~mm}$ outer diameter (World Precision Instruments, Sarasota, FL) were pulled to tip sizes of $\sim 0.5 \mu \mathrm{m}$ with a Sutter P-97 pipette puller (Sutter Instruments, Novato, CA) and stored on ice. Tubulin was back-loaded into injection pipettes using microloader pipettes (Eppendorf). The cell soma was injected by means of an Eppendorf Microinjector 5242/Micromanipulator 5170 for $\sim 0.5-1.0 \mathrm{sec}$ with a pressure of $1.0-1.5 \mathrm{kPa}$. This injection time, pressure, and concentration of TMR-tubulin increased the concentration of tubulin within the injected neurons by roughly $2-5 \%$. On average $20 \%$ of the injected neurons within a dish remained viable, as evidenced by their ability to incorporate TMR-tubulin into MT polymer, maintain motility, and continue to extend processes. Neuronal viability was apparent almost immediately after injection, although most cells were not imaged until at least $1 \mathrm{hr}$ after injection to allow incorporation of tubulin into polymer. The locations of viable, injected neurons were recorded with a Newvicon video camera (Dage-MTI, Michigan City, IN) and video graphic thermal printer (Sony, Tokyo, Japan). The prints 
provided a permanent record of the location of the cells relative to landmarks on the etched coverslips. Dishes were returned to the incubator to allow the medium to warm and the $\mathrm{pH}$ to equilibrate. Just before time-lapse imaging, the coverslips containing the neurons were enclosed in a chamber consisting of a $15 \mathrm{~mm}$ (inner diameter) glass ring (Thomas Scientific, Swedesboro, NJ) on which was placed a 25 -mm-round coverslip (Fisher, Itasca, IL). The chambers were attached to the culture dishes with silicone grease (Goslin and Banker, 1991). Before the chamber was sealed with a coverslip, most of the medium was removed from the chamber, and the cells were rinsed twice with nitrogen-saturated (low oxygen) imaging medium-1 or imaging medium-2. The design of this chamber resulted in a low oxygen environment for the duration of the imaging sessions. This reduced the formation of free oxygen radicals, which are particularly injurious to neurons. For experiments involving imaging for extended time periods $(>2 \mathrm{hr})$, the chambers containing the cells in serum-free medium were sealed with a glass ring, coverslip, and silicone grease. Over time this decreased the amount of dissolved oxygen in the medium and thus maintained the health of the neurons (Brewer and Cotman, 1989).

Time-lapse imaging. Chambers containing injected neurons were placed on the stage of an Axiovert $135 \mathrm{~m}$ inverted microscope (Zeiss). The microscope was equipped for DIC and epifluorescence microscopy (Zeiss long bandpass rhodamine filter set). The microscope also had a Keller port to maximize the amount of emitted light reaching the camera. Both the $100 \mathrm{~W}$ halogen light source used for DIC imaging and the $100 \mathrm{~W}$ HBO mercury arc light source (AttoArc, Zeiss) used for epifluorescence were equipped with electronically controlled shutters (Uniblitz shutters, Vincent Associates, Rochester, NY) to reduce illumination of the neurons. To assess whether injected neurons were still viable and well labeled, a low-power image was taken under epifluorescence illumination with a $40 \times$ Plan-Neofluar/1.3 NA objective. All fluorescence images were projected through the Keller port to a slowscan liquid-cooled charge-coupled device (CCD) camera (Photometrics PXL, Tucson, AZ) equipped with a Kodak KAF-1400 chip. Illumination during all epifluorescence imaging was reduced to $10-25 \%$ of the output of the light source by placement of neutral density filters (Chroma Technology, Brattleboro, VT) in the light path. Cells were maintained at $36^{\circ} \mathrm{C}$ with an airstream incubator (Nicholson Precision Instruments, Bethesda, MD).

Well labeled, motile neurons were imaged in time lapse with a $100 \times /$ 1.3 NA Fluar objective (Zeiss). Images were acquired every 10-20 sec, with 100-1000 msec exposures, under the low light level conditions described above. For experiments involving imaging for extended time periods, images were taken every $2-4 \mathrm{hr}$, and chambers were returned to the incubator between times of imaging. Because fluorescence tended to fade over time, it was often necessary to increase the exposure time during imaging. This sometimes resulted in higher background levels in images acquired at later time points in a given sequence. Fine focus was manually controlled with an LEP MAC 2000 focus controller (Ludl Electronic Products, Hawthorne, NY). The CCD camera and shutters were controlled by Metamorph 2.5 software (Universal Imaging, West Chester, PA) running on a Pentium-based computer (Datastor, Boulder, $\mathrm{CO}$ ). Images were collected at a $500-800 \mathrm{kHz}$ transfer rate, either unbinned or binned $(2 \times 2)$. The slow transfer rate and the inherent low dark current of the Kodak CCD resulted in images containing very low noise, whereas binning increased the sensitivity of the camera, resulting in shorter exposures. Unbinned images $\left(6.8 \mu \mathrm{m}^{2}\right.$ pixel size of CCD, $3.8 \times$ oversampling) met the Nyquist criterion for sampling, whereas binned images (13.6 $\mu \mathrm{m}^{2}$ effective pixel size of CCD, $1.9 \times$ oversampling) did not meet this sampling criterion (Inoué, 1986; Inoué and Spring, 1997). However, at low light levels resolution is often limited by too few photons reaching the detector, so binning resulted in improved image quality (Salmon et al., 1998). All images were saved to the hard drive in 12-bit format. To determine the amount of TMR-tubulin incorporation into the MT array of injected neurons, free tubulin was extracted with MT stabilizing buffer (Yu et al., 1996) containing 0.1\% Triton X-100 (Sigma) for 5 min. Cells were fixed for 15 min with $4 \%$ paraformaldehyde $/ 0.25 \%$ glutaraldehyde in MT stabilizing buffer.

Immunocytochemistry. Images of living cortical neurons with large paused growth cones were acquired with phase optics $(20 \times / 0.5 \mathrm{NA})$ and a cooled CCD camera (Princeton Instruments MicroMax). Cultures were immediately extracted and fixed as above. After cultures were washed briefly in PBS, they were blocked in 5\% normal donkey serum (NDS)/ PBS for $1 \mathrm{hr}$ and incubated with a Cy3-coupled anti- $\beta$-tubulin antibody (Sigma) diluted 1:100 in 5\% NDS/PBS. Cells were mounted in a glycerol/PBS solution containing $25 \mathrm{mg} / \mathrm{ml}$ 1,4-diazabicyclo-octane (Sigma) and $4 \mathrm{mg} / \mathrm{ml} \mathrm{n}$-propyl gallate (Sigma). Fluorescent images of growth cones were acquired with a $100 \times / 1.3$ NA Plan Neofluor objective (Zeiss) and a cooled CCD camera.

Measurements and data analysis. MT movements were discernable by playing back time-lapse images as a movie or frame by frame. The same MT could usually be followed through sequential frames because individual MTs did not drastically alter their sizes or shapes from one frame to the next. However, to determine more objectively the distance and direction of MT movement, an additional method of frame-by-frame analysis was used. This method, which has been used to track movements of intracellular organelles and particles with DIC microscopy (Weiss and Maile, 1993; Russ 1995), consisted of subtracting time-lapse images from one another in sequence. Subtracting one image from a previously collected image produced a composite image in which the region that had contained an individual MT appeared black and the region to which the MT had moved appeared white. All regions in which no MT movement
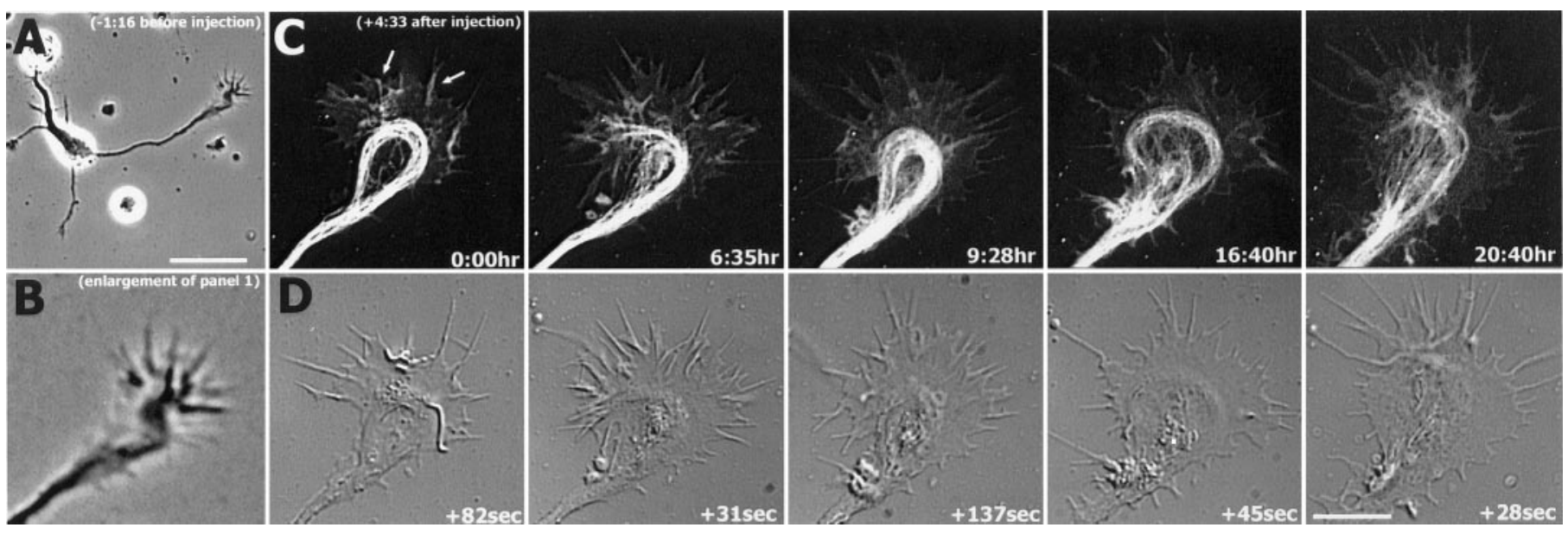

Figure 2. Formation of a MT loop in the central region of a large paused growth cone. A low-power phase image of a cortical neuron $(A)$, taken 1 hr and 16 min before injection with TMR-tubulin, is shown. The neuron has extended an axon with a prominent terminal growth cone. $B$, An enlarged image of the growth cone in $A$ shows that MTs (phase dark band in growth cone) are beginning to curve during the initial formation of the MT loop. $C$, A series of fluorescent images of the same growth cone shown in $A$ and $B$ after injection of TMR-tubulin into the neuron. Note the prominent MT loop at 0:00 hr and the short MTs (arrows) throughout the peripheral lamellipodium. The loop uncurls and reforms several times over a period of $20 \mathrm{hr}$, without elongation of the axon. In the matching DIC images $(D)$, vesicles and mitochondria remain within the loop. Scale bar: $A$, $30 \mu \mathrm{m}$; $B-D, 10 \mu \mathrm{m}$. 

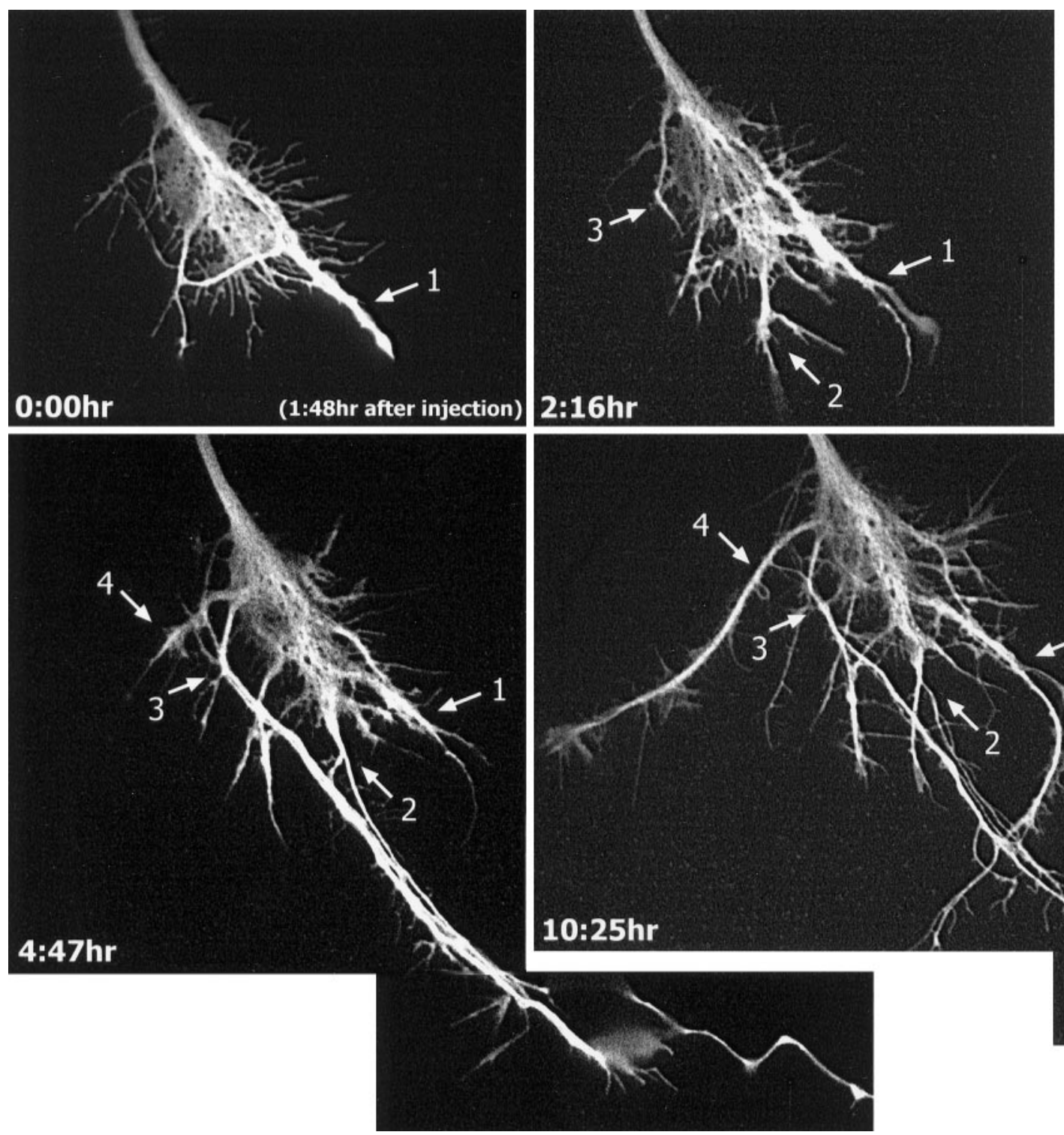

Figure 3. Invasion of MTs into multiple branches extending from a large paused growth cone. At 0:00 hr a large paused growth cone is beginning to extend a small process filled with MTs (1). Several hours later (2:16 hr ) MTs begin to invade two other small processes (2,3). At 4:47 hr these small processes $(2,3)$ have extended and are heavily invested with MTs. In another small process $(4,4: 47 h r)$ MTs invade distal regions. Almost 6 hr later $(10: 25 h r)$, both processes 1 and 4 have extended to form prominent branches. Note that the concentration of MTs in the branches increases, whereas the concentration of MTs in the paused growth cone decreases. Scale bar, $10 \mu \mathrm{m}$.

occurred appeared uniformly gray. As a further means of measuring MT movement, we took advantage of the fact that microinjection of small amounts of labeled tubulin often results in punctate labeling (speckling) of MTs within cells (Waterman-Storer and Salmon, 1998). This made it possible to distinguish actual MT movement from treadmilling, because if the speckles moved with the MT, then actual MT movement had occurred. However, if the speckles remained stationary while the MT appeared to move, then apparent MT movement was actually caused by treadmilling.

Instantaneous velocities of MT movements were calculated by dividing the movement of the bright marks between frames by the time-lapse interval. Average velocities were calculated by dividing the total amount of movement by the total time-lapse interval. Peak velocities were the fastest instantaneous velocity in a time-lapse series. All distances were calibrated in both the $\mathrm{x}$ and $\mathrm{y}$ directions by means of a stage micrometer (Graticules, Tonbridge, Kent, England). The pixel size of unbinned images corresponded to $0.068 \mu \mathrm{m}^{2}$, whereas the pixel size in binned $(2 \times$ 2) images corresponded to $0.136 \mu \mathrm{m}^{2}$. Well focused, individual MTs had minimum diameters of four pixels $(0.27 \mu \mathrm{m})$ in unbinned images and two pixels $(0.27 \mu \mathrm{m})$ in binned images, which is the diffraction-limited lateral resolution of the objective and wavelength of illumination used. All tracings of individual MTs presented in time-lapse images were two and four pixels in width for binned and unbinned images, respectively.

Levels of MTs at branch points and surrounding regions were determined by measuring the average pixel values in areas of the axon where a short $(<20 \mu \mathrm{m})$ interstitial branch had formed. Random 12-bit images were selected from the beginning, middle, and end of each time lapse series. Background levels of free tubulin were determined by measuring pixel values in areas of the growth cone devoid of MTs. These values were subtracted from each image in the series. Average pixel values were determined for $10 \mu \mathrm{m}$ regions proximal to, distal to, and directly at the branch point. These regions included the entire width of the axon to control for changes in the diameter of the axon at branch points. A ratio was computed for the average pixel value at the branch point with that of the surrounding regions.

Series of images in 12-bit format were archived onto CDs with a compact disk recorder (Pinnacle Micro Technology, Irvine, CA). Images were analyzed off-line using a Pentium-Pro-based workstation (Dell, Round Rock, TX) running Metamorph 3.5 software (Universal Imaging). All analyses of MT movement were performed on 12-bit images. All 

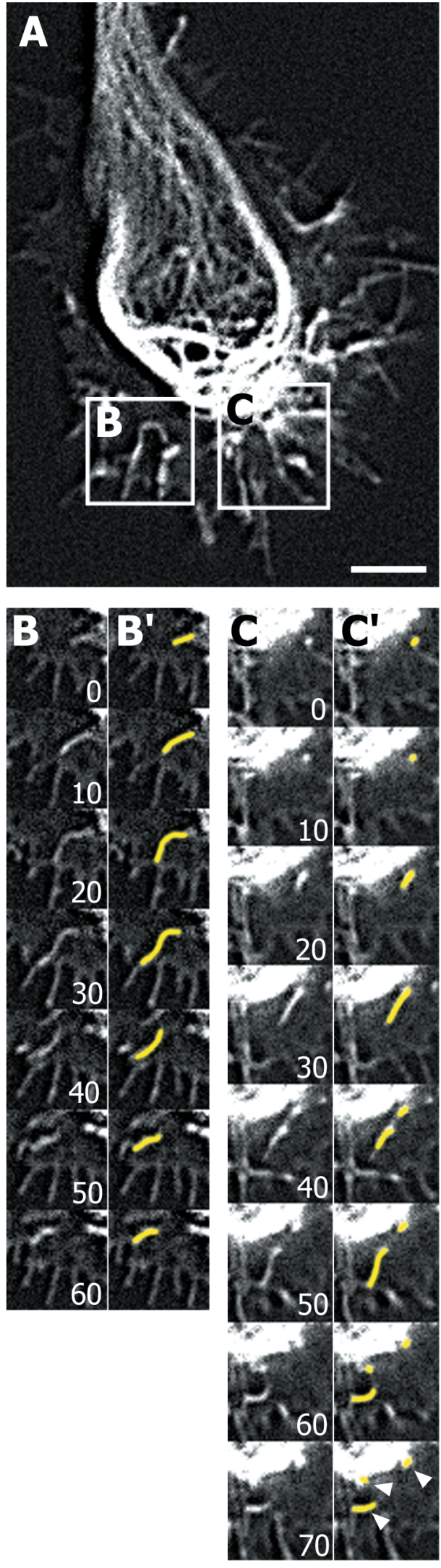

Figure 4. Movement and fragmentation of individual MTs in a paused growth cone. $A$, A large paused growth cone shows a prominent MT loop in the central region. MT movements shown in image sequences $B$ and $C$ occur in regions of the growth cone indicated by boxes. In sequence $B$ a MT elongates while moving rapidly into the peripheral lamellipodium figures were compiled from 8-bit images with Photoshop 5.0 (Adobe Systems, Mountain View, CA) and sharpened using the unsharp mask filter in either Metamorph or Photoshop. Graphing was performed using Sigmaplot 4.0 (SPSS, Chicago, IL), and statistical analysis was performed using Microsoft Excel (Redmond, WA).

\section{RESULTS}

To examine MT movements in growth cones and developing axon branches, we chose large pyramidal cortical neurons that develop obvious polarity in vitro (Kriegstein and Dichter, 1983; de Lima et al., 1997). By 2 d in culture, the neurons developed several short minor processes and a single long axon. The axon was tipped by a growth cone and usually extended prominent interstitial branches. We injected $>1500$ pyramidal neurons. Approximately $20 \%$ survived injection, and of these 98 were imaged for periods of $10 \mathrm{~min}$ to $2 \mathrm{hr}$ at intervals of every $10-20 \mathrm{sec}$. The data presented here were obtained from 44 sequences from 29 neurons. In additional cases, to study long-term branching events, neurons were imaged for longer time periods of up to $29 \mathrm{hr}$ after injection of tubulin. For these cases images were acquired at intervals of several hours. Because growth cone behaviors and branch formation were similar to those observed previously in uninjected cells (Szebenyi et al., 1998), it is unlikely that microinjection and imaging procedures disrupt growth cone motility and axon extension. In most cases, the fluorescent tubulin diffused to all regions of the neuron (excluding the nucleus) within 15 min of injection (Fig. 1). However, imaging of the neurons was begun $45 \mathrm{~min}$ to $2 \mathrm{hr}$ after injection, which was sufficient time for the fluorescent tubulin to become incorporated into most of the MT array [see, for example, Sabry et al. (1991)]. This resulted in brightly labeled MTs that were easily distinguishable against the low amounts of background fluorescence arising from unincorporated free tubulin.

To document MT movement it was important to establish criteria for distinguishing individual MTs and determining whether they moved. In large, flat, growth cone lamellipodia, individual MTs could usually be resolved because of their low density. In contrast, at axon branch points and in developing branches, MTs were more difficult to resolve. We therefore used the dimensions of MTs in growth cone lamellipodia as criteria for evaluating MTs as individuals at branch points (see Materials and Methods). In growth cones and developing branch points, movements of individual MTs were determined by playing back timelapse images (as described in Materials and Methods). If both ends of the MT moved at the same rate in the same direction in at least three consecutive frames, we classified the motion as MT movement.

Another important issue is whether changes in MT distribution result from motor-based movement of MTs or from treadmilling. Treadmilling in vivo involves coordinated disassembly at the minus end of the MT and assembly at the plus end. To distinguish between treadmilling and movement, we took advantage of the fact that incorporation of fluorescent tubulin does not always result in continuous labeling of the MT, especially when smaller

$(0-30 \mathrm{sec})$ and then shortens while moving laterally $(40-60 \mathrm{sec})$. In sequence $C$ a MT elongates $(0-30 \mathrm{sec})$ and then fragments into two shorter MTs (40 sec). The shorter MT segment remains stationary without elongating or shortening (50-70 sec), whereas the longer MT segment grows slightly $(50 \mathrm{sec})$ and then fragments a second time $(60-70 \mathrm{sec})$. Sequences $B^{\prime}$ and $C^{\prime}$ highlight in yellow the MT shown in $B$ and fragmented MT shown in $C$ (arrowheads in last frame point to three MT fragments). Scale bar, $5 \mu \mathrm{m}$. 

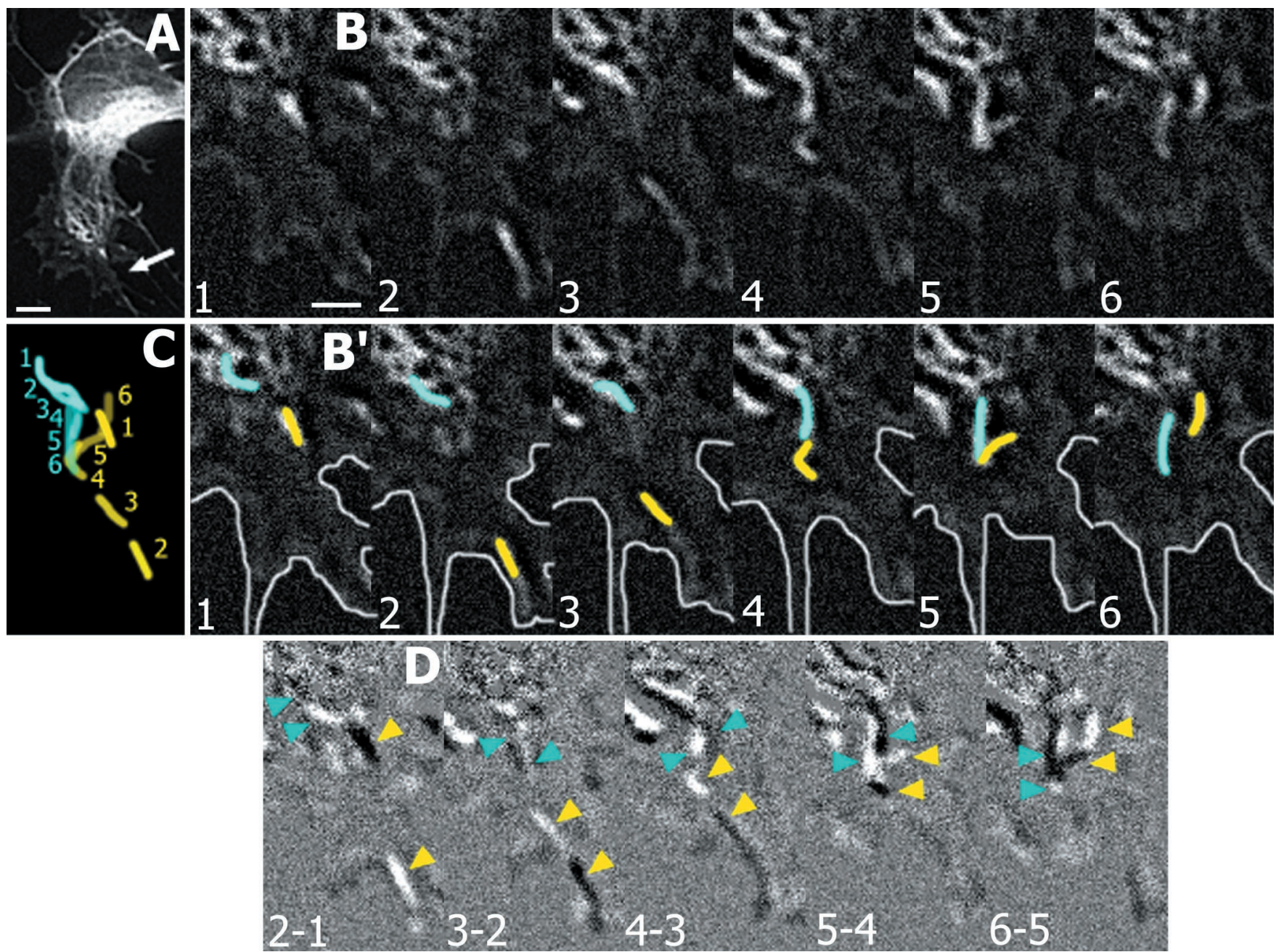

Figure 5. Individual MTs moving independently in all directions within a growth cone lamellipodium. Low-magnification image $A$ shows a growth cone that emerged from the cell body of a newly plated neuron that was just beginning to extend processes. Arrow indicates the lamellipodium shown at higher power in $B$ and $B^{\prime}$. During the sequence $(B)$, MTs move out of the central region of the growth cone and explore its periphery. In $B^{\prime}$ two of these MTs have been highlighted yellow or blue for emphasis. In $C$ the trajectories of both MTs are summarized. The numerals in $C$ refer to frame numerals in $B$ and $B^{\prime}$. The lamellipodium is outlined in white to show changes in shape during the sequence. In $D$ composite images resulting from subtraction of the time-lapse images in $B$ from one another in sequence are shown. The region that contained an individual MT in the first frame appears black, and the region to which the MT moved appears white. The yellow and blue arrows point to the positions of the MTs highlighted yellow and blue in $B^{\prime}$. All regions in which no MT movement occurred appear uniformly gray (see Material and Methods). Frame numerals correspond to 15 sec intervals. Scale bars: $A$, $5 \mu \mathrm{m} ; B-D, 2 \mu \mathrm{m}$.

amounts of labeled tubulin are injected (see Materials and Methods). Under these conditions, some MTs were continuously labeled but others were intermittently labeled, which resulted in occasional bright speckles. These speckles were useful because they can serve as fiduciary marks for distinguishing MT movement from treadmilling (Waterman-Storer and Salmon, 1998).

\section{MT reorganization and movements in the growth cone}

We chose for analysis motile but pausing growth cones that were typically large, thin, and flat. These features optimized visualization of MTs. In the central region of such growth cones, MTs formed loops characteristic of pausing or slowly growing axons (Tsui et al., 1984; Lankford and Klein, 1990; Sabry et al., 1991; Tanaka and Kirschner, 1991). Growth cones with looped MTs often paused for many hours. However, during transitions of the growth cones from quiescent to growth states, MTs underwent a dramatic reorganization. As shown in Figure 2, bundles of looped MTs within a large paused growth cone splayed apart and re- formed into the loop several times. Toward the end of the recording period (16-20 hr), most of the MTs were splayed apart as the growth cone began to extend. The matching DIC images show that during growth cone pausing, vesicles and organelles were confined to the central region of the growth cone within the MT loop. Imaging of additional growth cones $(n=10)$ for periods of 10-24 hr showed that during transitions from pausing to growth, MTs invariably reorganized from a looped to a splayed configuration. We also observed growth cones $(n=5)$ from which branches emerged directly. In the growth cone shown in Figure 3, branches began as filopodia-like processes (time 0:00 hr), some of which were invaded by MTs. Only those filopodial processes that contained MTs developed into branches, whereas those lacking MTs either disappeared or remained as filopodia. However, even longer branches tipped by growth cones and heavily invested with MTs were capable of regressing, suggesting that although MT invasion is necessary for development of branches, their presence 

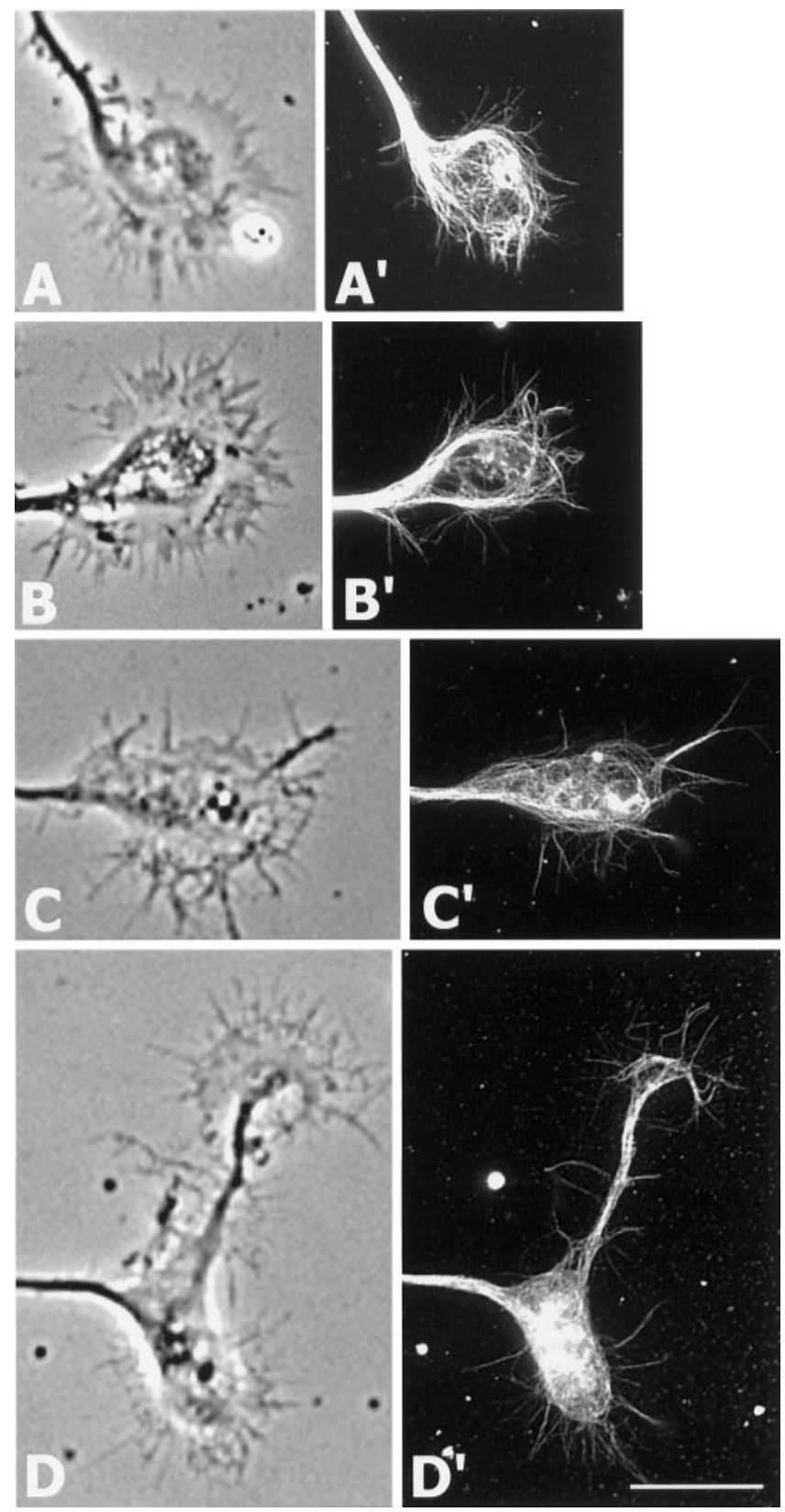

Figure 6. Organization of MTs in large paused growth cones revealed by immunocytochemistry. Phase images in $A-D$ were obtained from living growth cones. Fluorescent images in $A^{\prime}-D^{\prime}$ were obtained from the same growth cones after extraction of free tubulin, fixation, and immunostaining for $\beta$-tubulin. The fluorescent images in $A^{\prime}-C^{\prime}$ show that MTs form prominent loops in the central growth cone domain and that individual MTs extend into peripheral regions of the lamellipodia and into filopodia. $D$ shows a large, flat, branching region that resembles a growth cone on the axon shaft. The axon is also tipped by a growth cone. In both regions MTs penetrate into the lamellipodia and filopodia $\left(D^{\prime}\right)$. Scale bar, $20 \mu \mathrm{m}$.

does not necessarily guarantee the survival of a branch. The examples in Figures 2 and 3 were obtained from two different growth cones. However, we were also able to image single growth cones during the entire progression from pausing to branching. In every case $(n=10)$ MTs initially formed loops and then splayed apart followed by their invasion into newly forming branches that continued to extend.

To understand earlier stages of MT reorganization in growth cones, we imaged growth cones that were still undergoing pausing and contained looped MTs. As shown in Figure 4, the MT array formed a prominent loop in the central region of the paused growth cone. In several regions of the lamellipodium $(B, C)$, short dispersed MTs moved away from the looped MT bundle. Imaging of MTs at frequent intervals showed that MTs moved rapidly into the periphery of the lamellipodium $(B)$. As shown in $C$, an MT first elongated $(0-30 \mathrm{sec})$ and then fragmented $(30-50 \mathrm{sec})$. The longer section of the fragmented MT then itself fragmented into two MTs $(50-70 \mathrm{sec})$. In additional sequences $(n=9)$ of axonal growth cones (data not shown), short MTs were also observed moving away from the central MT bundle. Taken together, these results suggest that MT reorganization during the transitions to new growth may involve an initial breaking away of short MTs from the MT loop, followed by a splaying apart of the looped MTs and their entry into newly forming branches.

What other types of movement do MTs undergo in growth cones? We found that MTs explored lamellipodia with forward, backward, and lateral movements. Forward and backward movements occurred along axes radiating out from the central region of the growth cone, whereas lateral movements were perpendicular to these axes. Figure 5 shows a $75 \mathrm{sec}$ sequence of a growth cone that had emerged from the cell body of a newly plated neuron that was just beginning to extend processes. A short (2.5 $\mu \mathrm{m})$ MT (Fig. $5 B^{\prime}$, in yellow) advanced forward into the lamellipodium but after a few seconds abruptly changed direction and moved backward and laterally (Fig. $5 B, B^{\prime}, C$ ). The anterograde rate of movement of this $\mathrm{MT}$ was twice that of the retrograde rate. During this sequence, the MT did not grow or shrink but became momentarily kinked (Fig. 5B, $B^{\prime}, C$, frame 4 ). Plotting of the movements of a nearby 3.5- $\mu \mathrm{m}$-long MT (in blue) revealed that it moved in an entirely different and independent trajectory. The MT shown in blue moved laterally and anterogradely, whereas the MT shown in yellow moved retrogradely at a different angle. Movements of these MTs were confirmed independently using the image subtraction method (see Materials and Methods). As shown in Figure $5 D$, this method of analysis also showed that the MTs identified in $B$ moved in the trajectories indicated in $B^{\prime}$ and $C$. Similar analyses of MT movements in other growth cones $(n=9)$ showed that individual MTs were able to move independently of one another. For example, in one growth cone (data not shown), six separate MTs were followed for 20 min, during which two moved forward, two moved backward, and two moved laterally at different angles and at different rates (average velocities ranged from 4.2 to $8.6 \mu \mathrm{m} / \mathrm{min}$, and peak velocities ranged from 7.2 to $21.8 \mu \mathrm{m} / \mathrm{min}$ ).

The fluorescent MTs observed in these experiments were somewhat more difficult to focus along their lengths than those observed in previous studies on flatter non-neuronal cells (Keating et al., 1997), and therefore we wished to confirm independently their identity as MT polymers. To accomplish this, we compared the appearance of the microinjected neurons with the appearance of comparable cells that had been extracted to remove free tubulin and then immunostained to reveal the distribution of MTs. As shown in Figure 6, the distribution of MTs in the immunostained cells closely resembled the appearance of the cells injected with fluorescent tubulin. There is a prominent looped bundle of MTs within the growth cone, and in all cases MTs appeared within the lamellipodia and filopodia. Similar distributions of MTs were observed in growth cones of neurons that were not extracted to remove free tubulin and then stained with an antibody that recognizes only MT polymers, and also in 


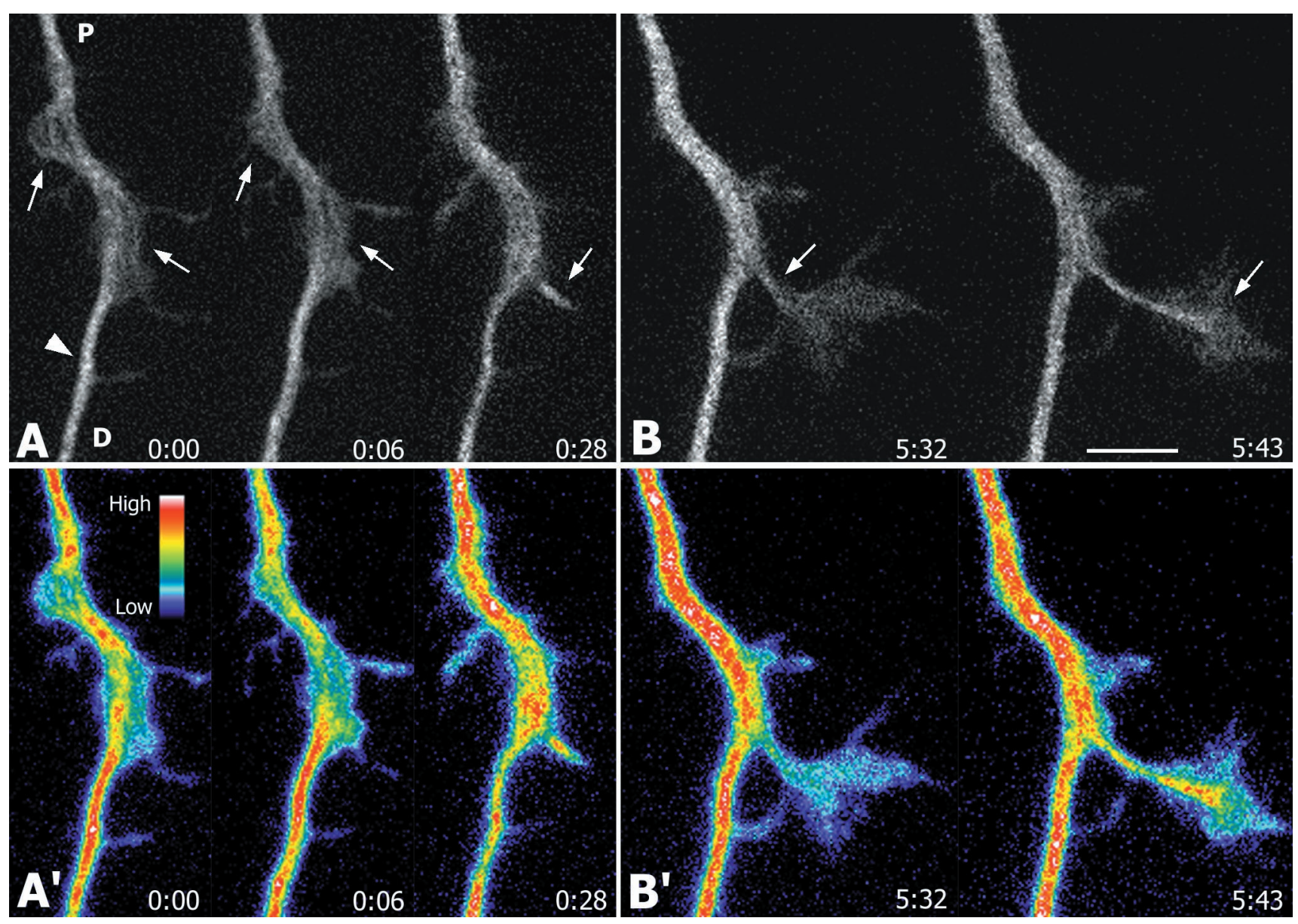

Figure 7. Splaying apart of the MT array within the axon shaft before interstitial branching. The sequence of black and white with matching pseudocolor images shows changes in the MT array before $\left(A, A^{\prime}\right)$ and during $\left(B, B^{\prime}\right)$ development of an interstitial branch. Pseudocolor images are shown to indicate fluorescent intensity from low to high (scale in $\left.A^{\prime}\right)$. Arrows in the first image $(A)$ point to two regions where MTs are splayed apart in comparison to the bundled array in the nonbranching region of axon shaft (arrowhead). The axon is labeled to indicate proximal $(P)$ and distal $(D)$ segments. Six minutes later the MTs in the upper region (arrow) have formed a bundle, whereas those in the lower region (arrow) remain splayed. At 28 min MTs have invaded a filopodial process (arrow). Five hours later $(B)$ MTs invade an interstitial branch elongating in the position of the filopodium (arrow in $A, 0: 28$ ). Arrows in both frames in $B$ indicate distal ends of the MTs. Time is shown in hours and minutes. Scale bar, $5 \mu \mathrm{m}$.

transmission electron micrographs of unextracted neurons (data not shown). These studies confirm that the fluorescent structures observed in our live-cell analyses correspond to MTs.

\section{MT reorganization and movements during axon branch formation}

In a previous study we found that interstitial branches developed from expanded regions of the axon shaft that resemble flat lamellipodia (Szebenyi et al., 1998). We focused on these regions to observe MT reorganization and movements at axon branch points. Observations at early stages of branch formation revealed disruptions in the bundled MT array. Figure $7 A$ shows several extensions forming along the axon shaft. In the first frame, MTs within the uppermost expanded region of the axon splayed apart (upper arrow). Six minutes later the MT array coalesced (upper arrow). However, at the same time MTs in the lower expanded region of the axon remained splayed (lower arrows), and at 28 min they began to coalesce and explore a filopodial process extending from the axon (arrow). At $5.5 \mathrm{hr}$ later (Fig. 7B), a branch tipped by a growth cone had developed from the region in which the MT discontinuity was previously observed, and short MTs had penetrated into the growing axon branch. At a subsequent time point (Fig. $7 B, 5: 43$ ), MTs continued to invade the elongating branch. At other developing axonal branch points, splaying apart of the MT array was correlated with development of a branch in 11 of 13 cases $(85 \%)$. Conversely, in cases where filopodia developed along the axon shaft but MTs within the axon remained bundled, branches never formed, although MTs frequently penetrated into these transient filopodia $(n=5)$. During branch formation, MTs, in addition to splaying apart, also exhibited a local breakdown and loss of polymer. At 11 of 13 axon branch points $(85 \%)$, levels of MTs were lower $(81 \pm 4.3 \%$, mean \pm SEM, $n=13$; $p<0.05, t$ test $)$ than regions of the axon proximal or distal to the branch point. However, in axon regions where MTs only transiently splayed apart without the loss of polymer $(n=18)$, branches never developed. These results suggest that development of branches from the axon shaft involves local splaying apart of the MT bundle accompanied by breakdown of MTs, a reorganization of MTs similar to that observed during formation of branches from paused growth cones.

MTs invaded developing branches from the axon shaft and moved forward and backward within them. Movements of MTs were often accompanied by their growth and shrinkage. However, analysis of 36 MTs in 20 growth cones and axon branches showed that during the time period of observation length changes in MTs 

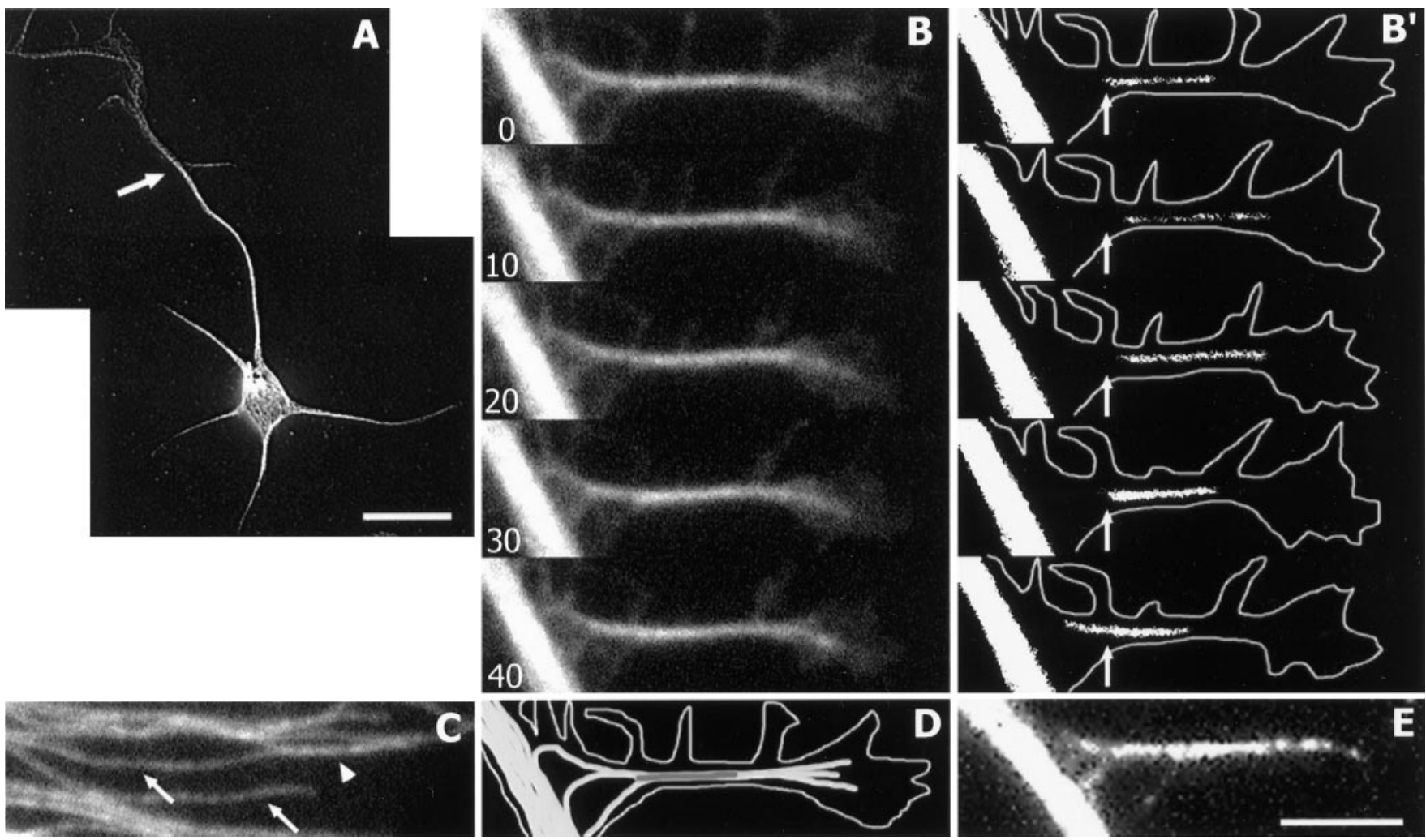

Figure 8. Retrograde movement of a MT in an axon branch. A low-magnification image is shown of a microinjected neuron after extraction and fixation $(A)$ to show the amount of fluorescent tubulin incorporated into MTs. The position of the interstitial axon branch imaged in $B$ is indicated by the arrow. Between 0 and $10 \mathrm{sec}(B)$ a MT elongates and moves anterogradely in the branch. Between 20 and $40 \mathrm{sec}(B)$ the MT shortens and moves retrogradely toward the brightly labeled axon shaft. The sequence of images in $B^{\prime}$ shows the MT moving in $B$. These images have been prepared by maximizing the contrast and minimizing the brightness of the images in $B$ to bring out the MT of interest. The branch has been outlined in white. Arrows in $B^{\prime}$ indicate a common reference point. MTs in a living fibroblast $(C)$ have morphologies and dimensions similar to those imaged in the axon branch $(B)$. Arrows in $C$ point to single MTs, and the arrowhead points to a bundle of MTs. In $D$, MTs in the branch were traced in white at time $0(B)$ and the same MT shown in $B^{\prime}$ is traced in gray. In $E$ the presence of MTs in this axon branch after extraction and fixation confirms that the fluorescent structures imaged in $B$ and traced in $D$ are actual MTs. Time is shown in seconds. Scale bars: $A, 20 \mu \mathrm{m} ; B-E, 5 \mu \mathrm{m}$.

were relatively small in comparison to the actual distances over which MTs moved. For example, in the branch shown in Figure 8, the MT grew longer during the first $20 \mathrm{sec}$. Between 20 and $40 \mathrm{sec}$ the MT moved retrogradely while also shortening. Because polymerization does not take place at the proximal (minus) ends of MTs in neurons (Baas and Ahmad, 1992), the MT must have been moving retrogradely in combination with shortening at the distal end. The actual movement of this MT was primarily retrograde at a peak rate of $\sim 12 \mu \mathrm{m} / \mathrm{min}$. However, in other branches, MTs moved primarily in the anterograde direction (data not shown). In one case (Fig. 9) a MT that was at least $20 \mu \mathrm{m}$ long was observed as it retreated at a rate of $0.5 \mu \mathrm{m} / \mathrm{min}$ from a stable axon branch that maintained growth cone motility and did not retract. However, the MT folded back on itself within the flattened branch point, presumably because it had difficulty re-entering the axon shaft. This observation demonstrates that even longer MTs can retreat from growing axon branches. In this case, even if the fluorescent speckles were not all on the same MT, all of the fiduciary marks were moving retrogradely at the same rate, thus demonstrating retrograde MT movement.

Imaging of branch formation along the axon shaft over extended time periods revealed that MTs undergo continual redistribution during the extension of some processes and the regression of others. For example, in Figure 10, MTs initially invaded several filopodia extending from a pausing growth cone. An axon branch extending just proximal to the growth cone was also heavily invested with MTs, and over the next several hours this MT-filled branch continued to extend. However, by the end of the recording session (at 10:27 hr), this branch had lost many of its MTs, became greatly attenuated, and retracted slightly. In contrast, several processes that began as thin filopodia subsequently developed into thick MT containing branches extending from the growth cone. In another sequence (Fig. 11), a large paused growth cone initially emitted a thin filopodial process as well as a new brightly fluorescent axon tipped by a growth cone. By $6 \mathrm{hr}$ later, the axon and its MTs had retracted, but the filopodium had developed into a prominent branch containing many MTs. Thus in these and other cases $(n=8)$, we found that growth of some processes accompanied by invasion of MTs often occurred simultaneously with the regression of other processes concomitant with a loss of MTs. It is possible that anterograde and retrograde MT transport, in addition to MT polymerization and depolymerization, play a role in directing MTs toward branches favored for growth and away from processes that retract.

In non-neuronal cells MTs can treadmill by adding and subtracting subunits to their plus and minus ends, respectively (Rodionov and Borisy, 1997; Waterman-Storer and Salmon, 1997). We are confident that we have observed MT movement for the following reasons. First, axonal MTs add subunits only at their plus ends (Baas and Ahmad, 1992), which are directed away from 

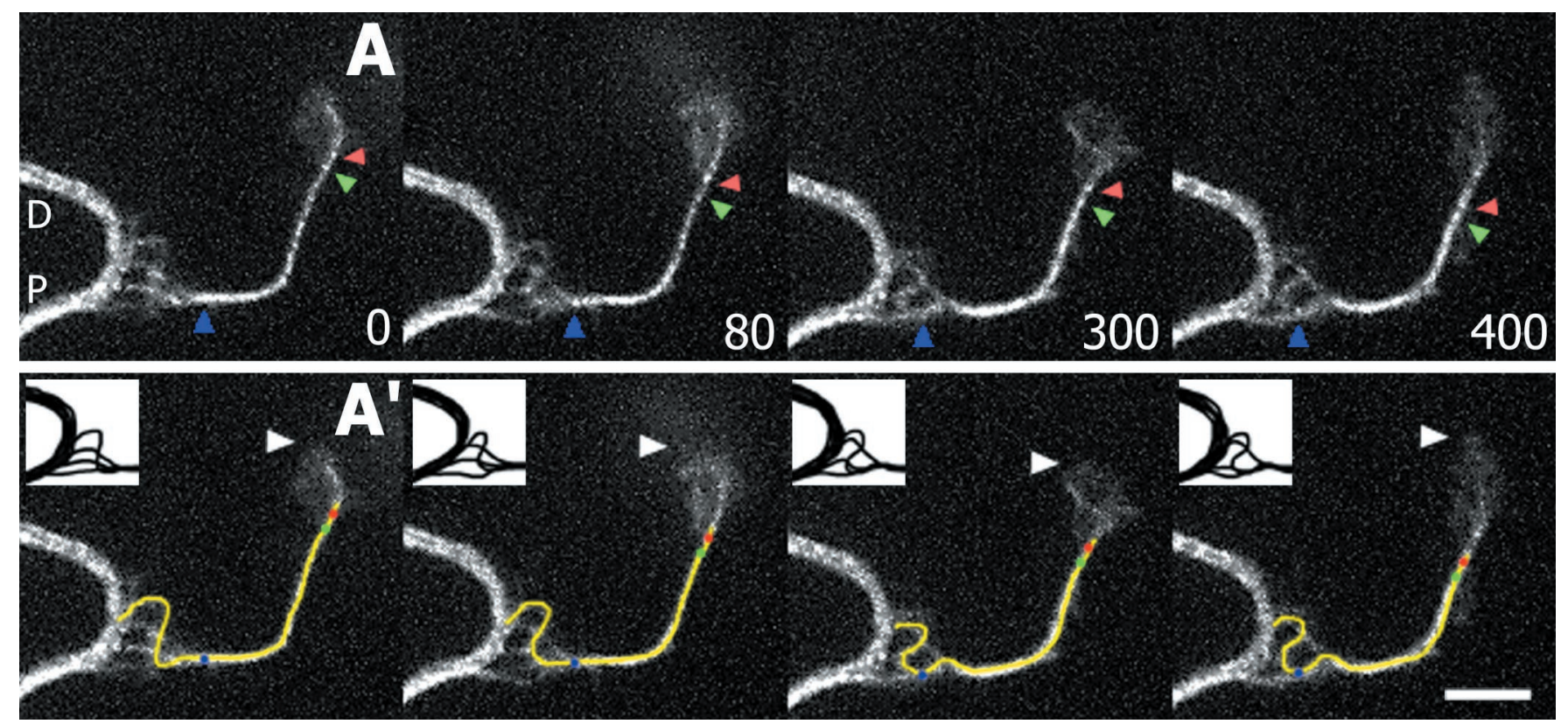

Figure 9. Withdrawal of a long MT from an axon branch. During this sequence a long MT within an axon branch moves retrogradely toward the axon shaft and folds back on itself in the expanded region of the branch point. In $A$, bright fluorescent speckles (indicated by arrowheads) at proximal and distal locations on the MT were used as fiduciary marks to follow retrograde movement. In $A^{\prime}$, the MT was traced in yellow, and the fluorescent speckles were marked with red, green, and blue dots. The tracings in $A^{\prime}$ show the distribution of the MTs in the proximal region of the branch. The distal tip of the growth cone (indicated by the arrowhead in $A^{\prime}$ ) exhibits motility and does not retract, showing that the MT is withdrawing from a stable branch. $P$ and $D$ refer to proximal and distal segment of the axon, respectively. Time is shown in seconds. Scale bar, $5 \mu \mathrm{m}$.

the cell body. Thus, the retrograde movements of MTs (with their minus ends leading) cannot be explained by polymerization. Second, treadmilling is unlikely to occur in neurons because the minus ends of neuronal MTs are quite stable against disassembly (White et al., 1987). Third, we found that MTs are able to change their direction of movement with great rapidity, which is inconsistent with MT treadmilling. Finally and most importantly, we carefully monitored fluorescent fiduciary marks produced by discontinuous labeling of the MTs and found that these marks moved in concert with the movement of the MTs.

\section{Rates of MT movement}

Our overall impression was that shorter MTs had more complex and rapid movements. We therefore measured average and peak rates of MT movement in axonal growth cones and branches. As shown in Figure $12 A-C$, MTs moving anterogradely, retrogradely, and laterally had similar respective mean average velocities of $8.6 \pm 1.9,6.2 \pm 1.0$, and $7.5 \pm 0.6 \mu \mathrm{m} / \mathrm{min}$ and similar mean peak velocities of $14.5 \pm 2.9,13.0 \pm 1.8$, and $11.5 \pm 2.5$ $\mu \mathrm{m} / \mathrm{min}$ (all velocities \pm SEM statistically similar, $p>0.30$, single-factor ANOVA). Individual MTs sometimes moved at constant rates but others exhibited saltatory movements, changing speed and direction within seconds and accelerating rapidly to velocities up to $30.1 \mu \mathrm{m} / \mathrm{min}$. As shown in Figure $12 \mathrm{~B}$, MTs longer than $10 \mu \mathrm{m}$ tended to move at significantly slower rates than those that were shorter than $10 \mu \mathrm{m}$. Frame-by-frame analysis (20-100 frames) of the movements of three 14- to 19- $\mu$ m-long MTs showed that their rates of movement ranged from 0.14 to $0.47 \mu \mathrm{m} / \mathrm{min}$, respectively $(0.35 \pm 0.10$, mean $\pm \mathrm{SEM})$, and that they tended to move in a single direction without changing speed. As shown in Table 1, the mean average and mean peak velocities of MTs in growth cones and axon branches were similar. Rates of MT movement were also independent of the substrate on which the neurons were plated. On laminin or concanavalin-A sub- $\overline{\text { Table 1. Rates of MT movement in different regions of cortical neurons }}$

\begin{tabular}{cclc} 
& $\begin{array}{l}\text { Mean average } \\
\text { velocity }(\mu \mathrm{m} / \mathrm{min})\end{array}$ & $\begin{array}{l}\text { Mean peak veloc- } \\
\text { ity }(\mu \mathrm{m} / \mathrm{min})\end{array}$ & $\begin{array}{l}\text { Mean length } \\
\text { of MT }(\mu \mathrm{m})\end{array}$ \\
\hline Growth cone & $7.7 \pm 0.9(14)$ & $14.1 \pm 1.8(14)$ & $1.6 \pm 0.2(14)$ \\
& {$[4.2-17.1]$} & {$[6.6-25.5]$} & {$[0.5-3.0]$} \\
Axon branch & $8.2 \pm 1.4(14)$ & $13.5 \pm 2.3(14)$ & $4.2 \pm 1.3(14)$ \\
& {$[0.5-21.8]$} & {$[0.5-30.1]$} & {$[1.0-19.0]$}
\end{tabular}

All velocities and lengths are mean \pm SEM. The number of sequences in which MT movement was analyzed is in parentheses. The ranges of velocities and lengths of MTs are in brackets. All means were statistically similar $(p>0.05, t$ test).

strates, the mean average velocity was $7.2 \pm 1.0 \mu \mathrm{m} / \mathrm{min}(n=22$, mean \pm SEM $)$ and $6.9 \pm 0.8 \mu \mathrm{m} / \mathrm{min}(n=9$, mean $\pm \mathrm{SEM}, p>$ $0.8, t$ test), respectively. These results show that MTs move at similar rates in both terminal growth cones and developing axon branches and that short MTs move rapidly, whereas long MTs move slowly at relatively constant velocities.

\section{DISCUSSION}

The present study demonstrates that different stages of growth cone advance are correlated with different configurations of MTs. During pausing, growth cones consistently displayed a looped configuration of tightly bundled MTs, which is consistent with previous studies showing a strong correlation between the presence of MT loops and decreased rates of neurite outgrowth (Tsui et al., 1984; Lankford and Klein, 1990; Sabry et al., 1991; Tanaka and Kirschner, 1991). During the transition from a paused to an advancing growth cone, the MT loops splayed apart. Splaying was observed before the development of axon branches within the specific region of the axon shaft that subsequently gave rise to the branch. Similarities between the terminal growth cone and sites of branch formation should not be surprising, given that interstitial branches form from sites where the terminal growth cone had 

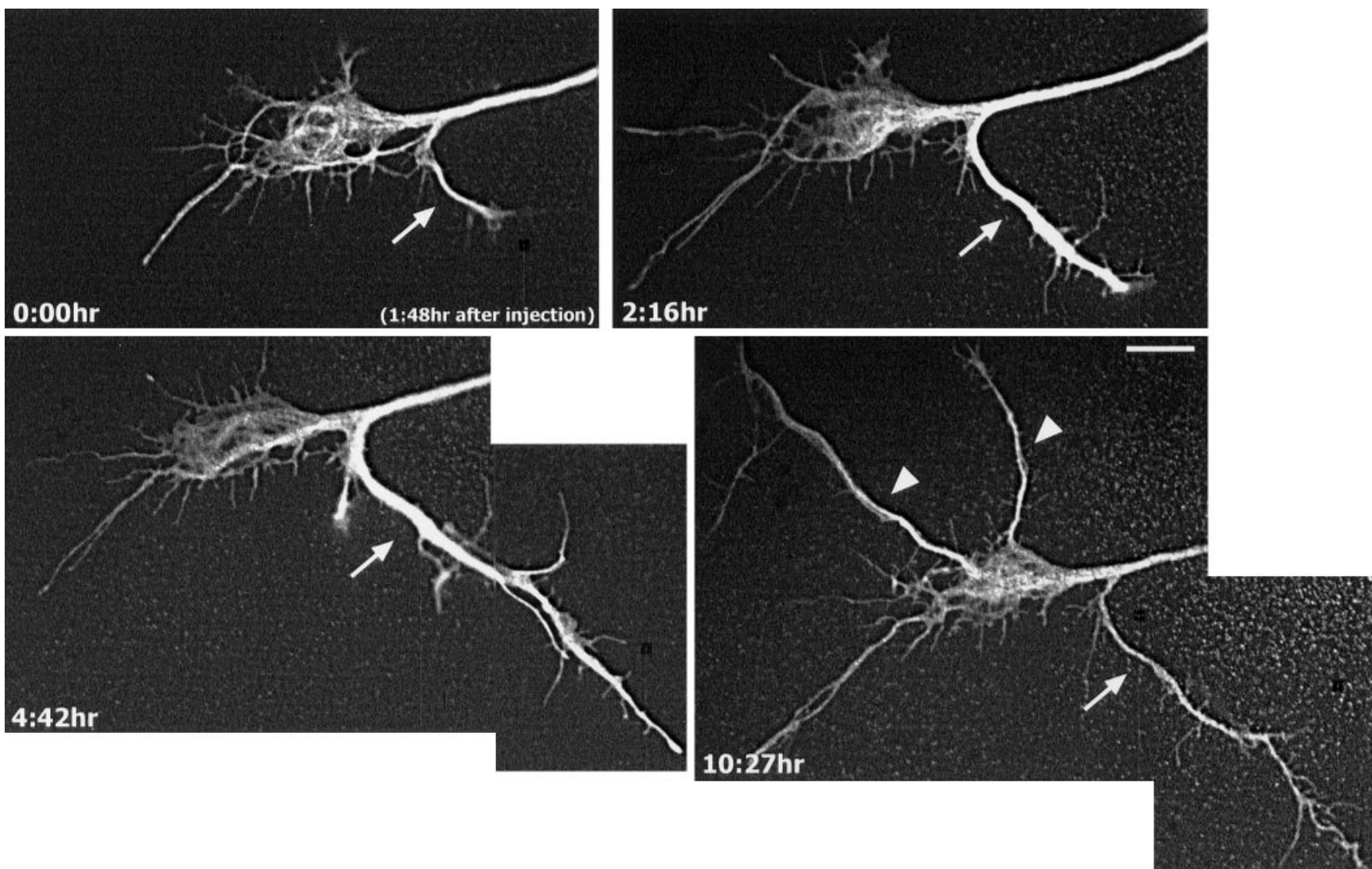

Figure 10. Withdrawal of MTs from an interstitial branch and proximal regions of a large terminal growth cone and invasion of MTs into two newly formed branches. At time 0:00 hr MTs form a loop in the paused growth cone and invade a newly formed interstitial branch (arrow). This interstitial branch elongates over the next $4 \mathrm{hr}$ (arrows at times 2:16hr and 4:42hr) as MTs continue to invest the branch. At 10:27 hr MTs withdraw from this interstitial branch (arrow), which has thinned and retracted slightly. Concomitantly, MTs invade two recently formed branches (arrowheads). Scale bar, $10 \mu \mathrm{m}$.

previously paused (Szebenyi et al., 1998). The similar MT behaviors in these two regions are probably important for enabling individual MTs to move more effectively into the lamellipodia and filopodia of growth cones and developing interstitial branches.

\section{Movement of MTs}

MT movement within neurons has been a controversial issue (Baas, 1997; Hirokawa et al., 1997), but the evidence appears to be mounting that MTs are indeed motile structures in neurons. Several recent studies have reported indirect experimental evidence that MTs are transported in axons (Slaughter et al., 1997; Ahmed et al., 1998) and into axon branches (Yu et al., 1996; Gallo and Letourneau, 1999), but these studies did not involve the direct visualization of MT movements. Our results demonstrate directly that MTs are indeed moving (and undergoing simultaneous length changes), at least in the two specialized regions of the axon that we examined. The denser packing of MTs and more cylindrical shape of the axon shaft precluded our ability to monitor these MTs for potential movement using the same methods that we used to study the more flattened growth cone and branch point regions. However, it seems reasonable that if MTs can move in these regions they can also move within the main shaft of the axon.

MTs typically moved in a direction parallel to their own long axis with one of the two ends leading, which strongly suggests that motor proteins engage individual MTs and generate forces that move them independently through the cytoplasm. The retrograde movement of MTs was unexpected, because MT movement in intact cells has thus far been documented only in the anterograde direction, when MTs move parallel to their own long axis (Terasaki et al., 1995; Keating et al., 1997). In several cases (data not shown) we did observe an MT, oriented parallel to the edge of a lamellipodium, moving backward toward the central region of the growth cone or axon shaft. In these cases, the MT moved in a direction roughly perpendicular to its long axis, rather than with one of the two ends leading. Such MT movements have been observed in other cell types and presumably result from a coupling of the MT to the actomyosin-based retrograde flow of cytoplasm (Waterman-Storer and Salmon, 1997). MTs that displayed this latter type of movement were not included in our analyses.

Another novel finding was that rates at which MTs moved were inversely proportional to their length. Short MTs of $10 \mu \mathrm{m}$ or less varied widely in their peak rates of movement $(4.6-30.1 \mu \mathrm{m} /$ min), whereas longer MTs moved at slow constant rates (0.14$0.47 \mu \mathrm{m} / \mathrm{min}$ ). Slower rates of movement by longer MTs are consistent with rates of $0.3-0.9 \mu \mathrm{m} / \mathrm{min}$ for slow axoplasmic transport as determined by pulse labeling in adult CNS axons (McQuarrie et al., 1986). The inverse relationship of MT length and transport rates may be attributable to more drag (Willard and Simon, 1983) on longer MTs. If this is correct, increasing the length of a MT by polymerization and further stabilizing it 

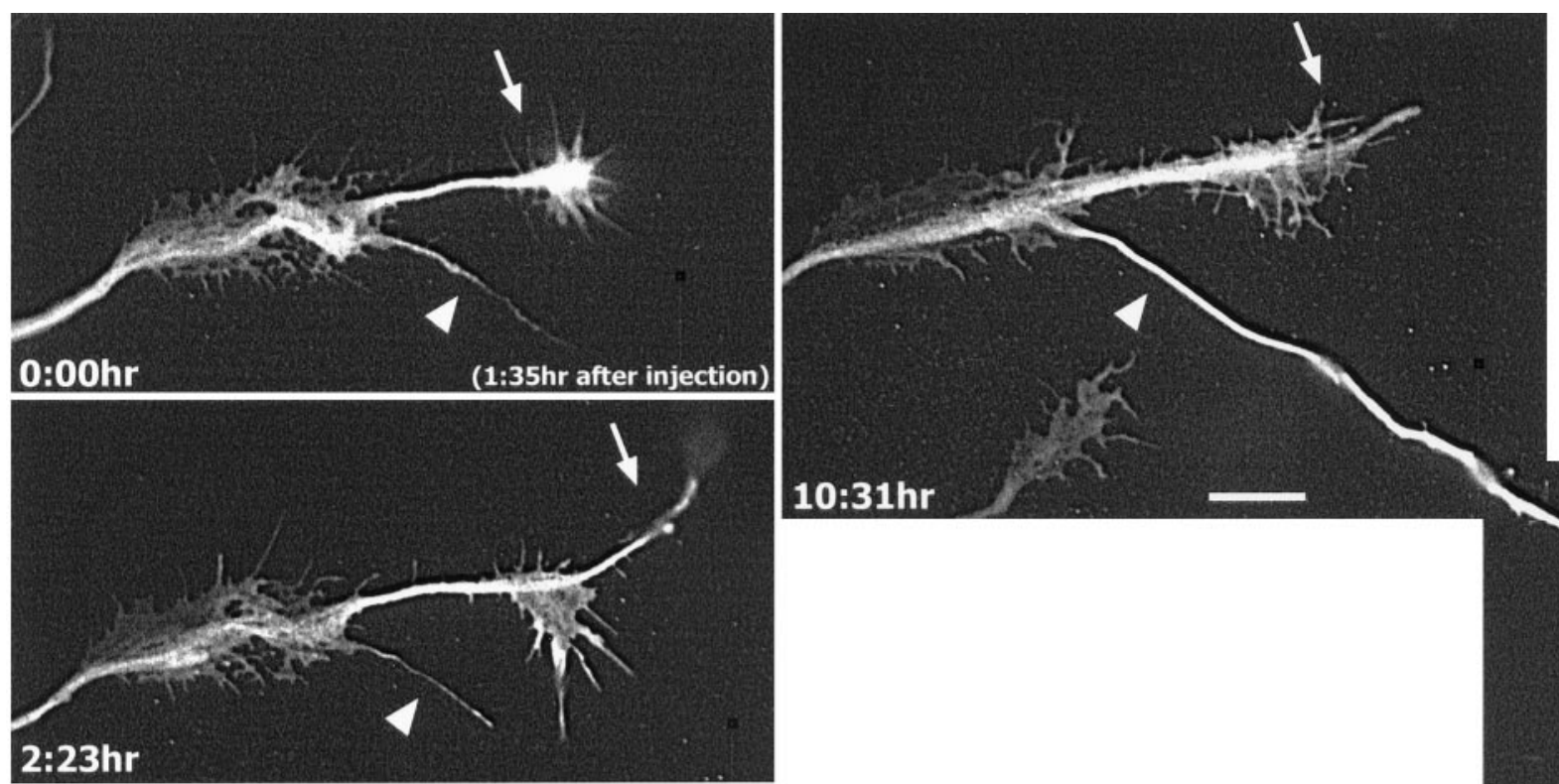

10:31hr
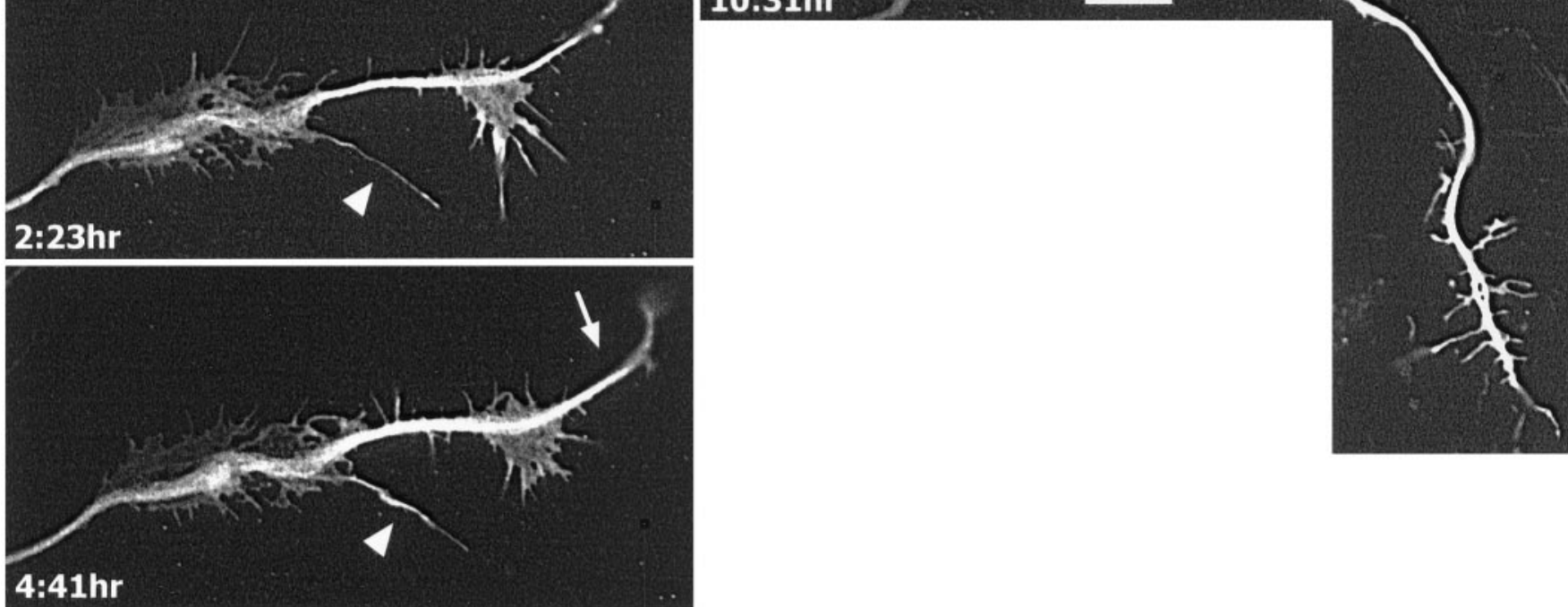

Figure 11. Redistribution of MTs in axon branches by invasion of some branches and withdrawal from others. A large paused growth cone ( $0: 00 h r)$ has extended a long filopodium (arrowhead) and a new axon tipped by a growth cone (arrow). By 2:23 hr the newly formed axon has continued to elongate (arrow), but the filopodium does not grow (arrowhead). Two hours later (4:41hr) MTs in the filopodium have increased (arrowhead), whereas the main axon has paused (arrow). Six hours later $(10: 31 \mathrm{hr})$ the filopodium has elongated into a prominent branch containing many MTs (arrowhead), whereas the axon and its MTs have retracted (arrow). Scale bar, $10 \mu \mathrm{m}$.

through interactions with MT-associated proteins (Desai and Mitchison, 1997) would result in slower MT movements. In contrast, in regions of new growth, short MTs undergoing more active movements may be required for rapid exploration of growth cones and developing branches. As MTs in these processes invade regions favored for growth, some of the MTs could then become stabilized in preferred directions by elongating and slowing down.

MT movements are commonly observed in cellular extracts from Xenopus eggs (Belmont et al., 1990; Heald et al., 1996), but relatively few reports have documented such movements within living cells. Keating and colleagues (1997), using methods similar to ours, showed MTs moving within epithelial cells. Interestingly, the rates of MT movement in the cellular extracts and within the epithelial cells were remarkably similar to the rates documented in the present study, suggesting that the mechanisms underlying MT movement may be highly conserved. Recent studies indicate that cytoplasmic dynein is a key motor protein that drives MT movement in cellular extracts (Heald et al., 1996) and in neurons (Ahmad et al., 1998). Similarities in the rates of anterograde and retrograde movement observed in the present study suggest that the same motor might be responsible for both types of movement. In theory, the forces generated by cytoplasmic dynein could result in the transport of MTs with their plus (Ahmad et al., 1998) or minus (Heald et al., 1996) ends leading (for review, see Baas,
1999). Alternatively, additional motors, such as the kinesinrelated motor known as Eg5, which is enriched in growth cones (Ferhat et al., 1998), might be responsible for some of the movements observed here.

\section{Reorganization of MTs}

Previous ultrastructural studies on cultured hippocampal neurons suggested that short MTs are important in growth cones and branch points and that longer microtubules fragment at branch points (Yu et al., 1994). Interestingly, the average length $(4.2 \mu \mathrm{m})$ of MTs at cortical axon branch points is similar to that of MTs at newly forming branches of hippocampal axons $(2.2 \mu \mathrm{m})$. We also observed short MTs averaging $1.6 \mu \mathrm{m}$ in length moving away from looped MT arrays in pausing growth cones, which is similar to the length observed in the growth cones of hippocampal neurons (Yu and Baas, 1994). Moreover, our studies confirm that MTs fragment. We observed directly a longer MT fragmenting twice into three short MTs within a pausing growth cone, which suggests that MT fragmentation may be a common mechanism for generating new MTs during transitions from quiescent to growth states. Fragmentation of longer MTs would result in a higher number of shorter MTs that would be ideally suited for rapid exploratory movements within growth cones and developing branches. After invasion into appropriate regions, these short 


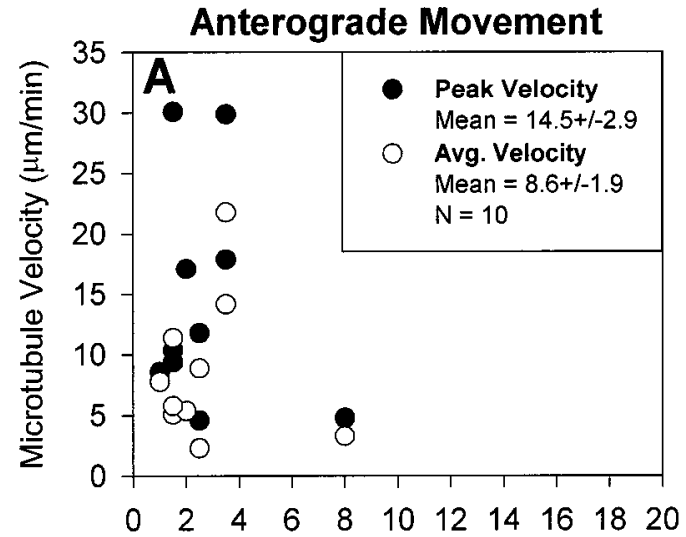

Average Microtubule Length $(\mu \mathrm{m})$

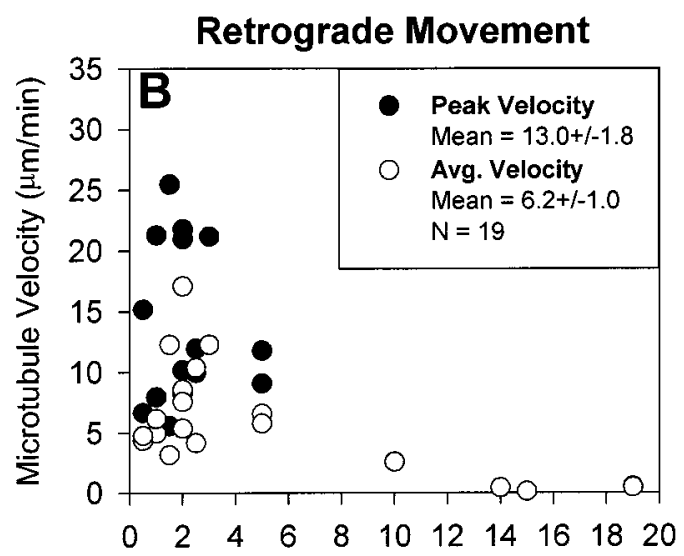

Average Microtubule Length $(\mu \mathrm{m})$

\section{Lateral Movement (GCs)}

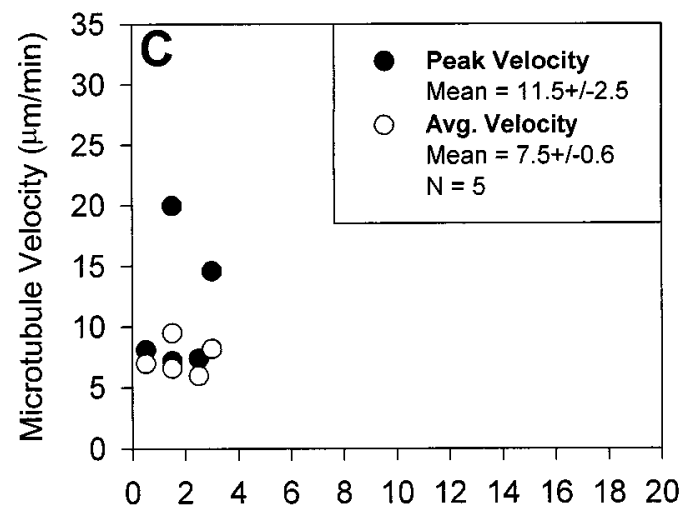

Average Microtubule Length $(\mu \mathrm{m})$

Figure 12. Similarity in rates of MT movement in anterograde, retrograde, and lateral directions. Average and peak rates of individual MT movement in growth cones and axonal branches were plotted as a function of MT length $(A-C)$. Rates of movement for shorter MTs $(<10 \mu \mathrm{m})$ were more rapid than for longer MTs $(>10 \mu \mathrm{m})$. All velocities are expressed as mean \pm SEM. Mean average velocities and mean peak velocities in anterograde, retrograde, and lateral directions are statistically similar ( $p>0.30$, single-factor ANOVA).
MTs could then elongate and become stabilized, allowing for further growth of the axon to occur.

Fragmentation of MTs in neurons may be locally regulated. For example, focal application of a calcium ionophore to axons of Aplysia neurons elicited new growth cones that developed into branched neuritic processes (Ziv and Spira, 1997). In these branching regions, the MT array appeared to be discontinuous, suggesting that locally induced MT fragmentation is correlated with growth of new axonal processes. In addition, a recent study (Gallo and Letourneau, 1998) showed that local application of NGF-coated beads initiated collateral sprouting of neurites and also caused localized debundling of MTs. What mechanisms might account for MT fragmentation in the axon? Several recent studies suggest that the protein katanin, known to have MTsevering properties in vitro (McNally and Vale, 1993; Hartman et al., 1998), is present in various cell types, including neurons (McNally and Thomas, 1998; Ahmad et al., 1999). One interesting possibility is that MT fragmentation may be regulated by intrinsic and/or extrinsic factors that locally activate katanin.

Might mechanisms other than severing by a protein such as katanin contribute to the generation of short MTs? In newt lung epithelial cells (Waterman-Storer and Salmon, 1997), MTs moving from the periphery toward the nucleus were observed to break because of bending and buckling. It is possible that buckling of MTs within loops or at developing interstitial branches could account for MT breakage and the generation of short MTs in growth cones. However, we did not observe buckling followed by breakage of MTs in any of our studies of growth cones or interstitial branches. Another possibility is that short MTs are generated de novo by local nucleation, as has recently been shown in certain non-neuronal cell lines such as PtK and 3T3 cells (Vorobjev et al., 1997; Yvon and Wadsworth, 1997). We cannot rule out this possibility, but the lower concentration of MTs at interstitial axon branch points makes this an unlikely mechanism for generating short MTs in these regions. Furthermore, earlier studies strongly suggested that local MT nucleation is suppressed in axons (Baas and Ahmad, 1992). It therefore seems reasonable to conclude that local fragmentation by a protein such as katanin is the principle means for generating short MTs observed in growth cones and at interstitial axon branch points.

It has long been recognized that in vivo developing cortical axons bypass their callosal or spinal targets before the development of an interstitial branch (O'Leary et al., 1988). After the branch projects toward the target, the region of the cortical axon distal to the branch degenerates (O'Leary et al., 1990). The present in vitro studies show a striking redistribution of MTs during the formation and withdrawal of axon branches. At present the mechanisms that regulate the long-term redistribution of MTs are unknown, but it is compelling to speculate that the kinds of anterograde and retrograde MT movements that we observed might be a key factor in determining whether an axon branch degenerates or continues to grow and stabilize.

\section{REFERENCES}

Ahmad FJ, Echeverri CJ, Vallee RB, Baas PW (1998) Cytoplasmic dynein and dynactin are required for microtubule transport into the axon. J Cell Biol 140:391-402.

Ahmad FJ, Yu W, McNally FJ, Baas PW (1999) An essential role for katanin in severing microtubules in the neuron. J Cell Biol 145:305-315. 
Baas PW (1997) Microtubules and axonal growth. Curr Opin Cell Biol 9:29-36.

Baas PW (1999) Microtubules and neuronal polarity: lessons from mitosis. Neuron 22:23-31.

Baas PW, Ahmad FJ (1992) The plus ends of stable microtubules are the exclusive nucleating structures for MTs in the axon. J Cell Biol 116:1231-1241.

Baas PW, Brown A (1997) Slow axonal transport: the polymer transport model. Trends Cell Biol 7:380-384.

Bastmeyer M, O'Leary DD (1996) Dynamics of target recognition by interstitial axon branching along developing cortical axons. J Neurosci 16:1450-1459.

Belmont LD, Hyman AA, Sawin KE, Mitchison TJ (1990) Real-time visualization of cell cycle-dependent changes in microtubule dynamics in cytoplasmic extracts. Cell 62:579-589.

Brewer GJ, Cotman CW (1989) Survival and growth of hippocampal neurons in defined medium at low density: advantages of a sandwich culture technique or low oxygen. Brain Res 494:65-74.

Chang S, Rodionov VI, Borisy GG, Popov SV (1998) Transport and turnover of microtubules in frog neurons depend on the pattern of axonal growth. J Neurosci 18:821-829.

de Lima AD, Merten MD, Voigt T (1997) Neuritic differentiation and synaptogenesis in serum-free neuronal cultures of the rat cerebral cortex. J Comp Neurol 382:230-246.

Desai A, Mitchison TJ (1997) Microtubule polymerization dynamics. Annu Rev Cell Dev Biol 13:83-117.

Dotti CG, Sullivan CA, Banker GA (1988) The establishment of polarity by hippocampal neurons in culture. J Neurosci 8:1454-1468.

Ferhat L, Cook C, Chauviere M, Harper M, Kress M, Lyons GE, Baas PW (1998) Expression of the mitotic motor protein Eg5 in postmitotic neurons: implications for neuronal development. J Neurosci 18:7822-7835.

Funakoshi T, Takeda S, Hirokawa N (1996) Active transport of photoactivated tubulin molecules in growing axons revealed by a new electron microscopic analysis. J Cell Biol 133:1347-1353.

Gallo G, Letourneau PC (1998) Localized sources of neurotrophins initiate axon collateral sprouting. J Neurosci 18:5403-5414.

Gallo G, Letourneau PC (1999) Different contributions of microtubule dynamics and transport to the growth of axons and collateral sprouts. J Neurosci 19:3860-3873.

Goslin K, Banker G (1991) Rat hippocampal neurons in low-density culture. In: Culturing nerve cells (Banker G, Goslin K, eds), pp 251281. Cambridge, MA: MIT.

Halloran MC, Kalil K (1994) Dynamic behaviors of growth cones extending in the corpus callosum of living cortical brain slices observed with video microscopy. J Neurosci 14:2161-2177.

Hartman JJ, Mahr J, McNally K, Okawa K, Iwamatsu A, Thomas S, Cheesman S, Heuser J, Vale RD, McNally FJ (1998) Katanin, a microtubule-severing protein, is a novel AAA ATPase that targets to the centrosome using a WD40-containing subunit. Cell 93:277-287.

Heald R, Tournebize R, Blank T, Sandaltzopoulos R, Becker P, Hyman A, Karsenti E (1996) Self-organization of microtubules into bipolar spindles around artificial chromosomes in Xenopus egg extracts. Nature 382:420-425.

Heidemann SR, Landers JM, Hamborg MA (1981) Polarity orientation of axonal microtubules. J Cell Biol 91:661-665.

Hirokawa N, Terada S, Funakoshi T, Takeda S (1997) Slow axonal transport: the subunit model of transport. Trends Cell Biol 7:383-388.

Hyman A, Drechsel D, Kellogg D, Salser S, Sawin K, Steffen P, Wordeman L, Mitchison T (1991) Preparation of modified tubulins. Methods Enzymol 196:478-485.

Inoué S (1986) Video Microscopy: the fundamentals. New York: Plenum.

Inoué S, Spring K (1997) Video microscopy: the fundamentals, Ed 2. New York: Plenum.

Keating TJ, Peloquin JG, Rodionov VI, Momcilovic D, Borisy GG (1997) Microtubule release from the centrosome. Proc Natl Acad Sci USA 94:5078-5083.

Kriegstein AR, Dichter MA (1983) Morphological classification of rat cortical neurons in cell culture. J Neurosci 3:1634-1647.

Kuang RZ, Kalil K (1994) Development of specificity in corticospinal connections by axon collaterals branching selectively into appropriate spinal targets. J Comp Neurol 344:270-282.
Lankford KL, Klein WL (1990) Ultrastructure of individual neurons isolated from avian retina: occurrence of microtubule loops in dendrites. Brain Res Dev Brain Res 51:217-224.

Letourneau PC (1996) The cytoskeleton in nerve growth cone motility and axonal pathfinding. Perspect Dev Neurobiol 4:111-123.

Lin CH, Forscher P (1993) Cytoskeletal remodeling during growth cone-target interactions. J Cell Biol 121:1369-1383.

McNally FJ, Thomas S (1998) Katanin is responsible for the M-phase microtubule-severing activity in Xenopus eggs. Mol Biol Cell 9:1847-1861.

McNally FJ, Vale RD (1993) Identification of katanin, an ATPase that severs and disassembles stable microtubules. Cell 75:419-429.

McQuarrie IG, Brady ST, Lasek RJ (1986) Diversity in the axonal transport of structural proteins: major differences between optic and spinal axons in the rat. J Neurosci 6:1593-1605.

Mikhailov AV, Gundersen GG (1995) Centripetal transport of microtubules in motile cells. Cell Motil Cytoskeleton 32:173-186.

Okabe S, Hirokawa N (1992) Differential behavior of photoactivated microtubules in growing axons of mouse and frog neurons. J Cell Biol 117:105-120.

Okabe S, Hirokawa N (1993) Do photobleached fluorescent microtubules move?: re-evaluation of fluorescence laser photobleaching both in vitro and in growing Xenopus axon. J Cell Biol 120:1177-1186.

O'Leary DD, Terashima T (1988) Cortical axons branch to multiple subcortical targets by interstitial axon budding: implications for target recognition and "waiting periods". Neuron 1:901-910.

O'Leary DD, Bicknese AR, De Carlos JA, Heffner CD, Koester SE, Kutka LJ, Terashima T (1990) Target selection by cortical axons: alternative mechanisms to establish axonal connections in the developing brain. Cold Spring Harb Symp Quant Biol 55:453-468.

Rodionov VI, Borisy GG (1997) Microtubule treadmilling in vivo. Science 275:215-218.

Russ JC (1995) The image processing handbook, Ed 2. Boca Raton, FL: CRC.

Sabry J, O'Connor TP, Evans L, Toroian-Raymond A, Kirschner M, Bentley D (1991) Microtubule behavior during guidance of pioneer neuron growth cones in situ. J Cell Biol 115:381-395.

Sabry J, O'Connor TP, Kirschner M (1995) Axonal transport of tubulin in Ti1 pioneer neurons in situ. Neuron 14:1247-1256.

Salmon ED, Shaw SL, Waterman-Storer CM, Maddox PS, Yeh E, Bloom K (1998) A high resolution multimode digital microscope system. In: Video microscopy (Sluder G, Wolf DE, eds), pp 186-214. San Diego: Academic.

Slaughter T, Wang J, Black MM (1997) Microtubule transport from the cell body into the axons of growing neurons. J Neurosci 17:5807-5819.

Suter DM, Errante LD, Belotserkovsky V, Forscher P (1998) The Ig superfamily cell adhesion molecule, apCAM, mediates growth cone steering by substrate-cytoskeletal coupling. J Cell Biol 141:227-240.

Szebenyi G, Callaway JL, Dent EW, Kalil K (1998) Interstitial branches develop from active regions of the axon demarcated by the primary growth cone during pausing behaviors. J Neurosci 18:7930-7940.

Takeda S, Funakoshi T, Hirokawa N (1995) Tubulin dynamics in neuronal axons of living zebrafish embryos. Neuron 14:1257-1264.

Tanaka EM, Kirschner MW (1991) Microtubule behavior in the growth cones of living neurons during axon elongation. J Cell Biol 115:345-363.

Tanaka E, Sabry J (1995) Making the connection: cytoskeletal rearrangements during growth cone guidance. Cell 83:171-176.

Tanaka E, Ho T, Kirschner MW (1995) The role of microtubule dynamics in growth cone motility and axonal growth. J Cell Biol 128:139-155.

Terasaki M, Schmidek A, Galbraith JA, Gallant PE, Reese TS (1995) Transport of cytoskeletal elements in the squid giant axon. Proc Natl Acad Sci USA 92:11500-11503.

Tsui HT, Lankford KL, Ris H, Klein WL (1984) Novel organization of microtubules in cultured central nervous system neurons: formation of hairpin loops at ends of maturing neurites. J Neurosci 4:3002-3013.

Vorobjev IA, Svitkina TM, Borisy GG (1997) Cytoplasmic assembly of microtubules in cultured cells. J Cell Sci 110:2635-2645.

Waterman-Storer CM, Salmon ED (1997) Actomyosin-based retrograde flow of microtubules in the lamella of migrating epithelial cells influences microtubule dynamic instability and turnover and is associated with microtubule breakage and treadmilling. J Cell Biol 139:417-434. 
Waterman-Storer CM, Salmon ED (1998) How microtubules get fluorescent speckles. Biophys J 75:2059-2069.

Waterman-Storer CM, Sanger JW, Sanger JM (1993) Dynamics of organelles in the mitotic spindles of living cells: membrane and microtubule interactions. Cell Motil Cytoskeleton 26:19-39.

Weiss DG, Maile W (1993) Principles, practice, and applications of video-enhanced contrast microscopy. In: Electronic light microscopy: techniques in modern biomedical microscopy (Shotton W, ed), pp 106-136. New York: Wiley Liss.

White LA, Baas PW, Heidemann SR (1987) Microtubule stability in severed axons. J Neurocytol 16:775-784.

Willard M, Simon C (1983) Modulations of neurofilament axonal transport during the development of rabbit retinal ganglion cells. Cell 35:551-559.

Yamamoto N, Higashi S, Toyama K (1997) Stop and branch behaviors of geniculocortical axons: a time-lapse study in organotypic cocultures. J Neurosci 17:3653-3663.

Yu W, Baas PW (1994) Changes in microtubule number and length during axon differentiation. J Neurosci 14:2818-2829.

Yu W, Ahmad FJ, Baas PW (1994) Microtubule fragmentation and partitioning in the axon during collateral branch formation. J Neurosci 14:5872-5884.

Yu W, Schwei MJ, Baas PW (1996) Microtubule transport and assembly during axon growth. J Cell Biol 133:151-157.

Yvon A-MC, Wadsworth P (1997) Non-centrosomal microtubule formation and measurement of minus end dynamics in A498 cells. J Cell Sci 110:2391-2401.

Ziv NE, Spira ME (1997) Localized and transient elevations of intracellular $\mathrm{Ca}^{2+}$ induce the dedifferentiation of axonal segments into growth cones. J Neurosci 17:3568-3579. 